Key Points:

- Nonlinear particle trapping in

velocity space by right-handed

parallel propagating electromagnetic

waves is studied in detail

-We determine the theoretical

pitch angle centroids for nominal

conditions in the upstream region

of Mars and Venus and planetary

foreshocks

- Similarities and differences in the particle's dynamics when forced by a circularly and elliptically polarized wave are analyzed

Correspondence to:

N. Romanelli,

Norberto.Romanelli@latmos.ipsl.fr

\section{Nonlinear Wave-Particle Interaction: Implications for Newborn Planetary and Backstreaming Proton Velocity Distribution Functions}

\author{
N. Romanelli ${ }^{1,2} \mathbb{( D}$, C. Mazelle ${ }^{1}(\mathbb{D})$, and K. Meziane ${ }^{3}(\mathbb{D}$ \\ ${ }^{1}$ Institut de Recherche en Astrophysique et Planétologie, University of Toulouse, CNRS, UPS, CNES, Toulouse, France, \\ ${ }^{2}$ Laboratoire Atmosphere, Milieux et Observations Spatiales, IPSL, CNRS, UVSQ, UPMC, Paris, France, ${ }^{3}$ Department of \\ Physics, University of New Brunswick, Fredericton, New Brunswick, Canada
}

\begin{abstract}
Seen from the solar wind (SW) reference frame, the presence of newborn planetary protons upstream from the Martian and Venusian bow shocks and SW protons reflected from each of them constitutes two sources of nonthermal proton populations. In both cases, the resulting proton velocity distribution function is highly unstable and capable of giving rise to ultralow frequency quasi-monochromatic electromagnetic plasma waves. When these instabilities take place, the resulting nonlinear waves are convected by the SW and interact with nonthermal protons located downstream from the wave generation region (upstream from the bow shock), playing a predominant role in their dynamics. To improve our understanding of these phenomena, we study the interaction between a charged particle and a large-amplitude monochromatic circularly polarized electromagnetic wave propagating parallel to a background magnetic field, from first principles. We determine the number of fix points in velocity space, their stability, and their dependence on different wave-particle parameters. Particularly, we determine the temporal evolution of a charged particle in the pitch angle-gyrophase velocity plane under nominal conditions expected for backstreaming protons in planetary foreshocks and for newborn planetary protons in the upstream regions of Venus and Mars. In addition, the inclusion of wave ellipticity effects provides an explanation for pitch angle distributions of suprathermal protons observed at the Earth's foreshock, reported in previous studies. These analyses constitute a mean to evaluate if nonthermal proton velocity distribution functions observed at these plasma environments present signatures that can be understood in terms of nonlinear wave-particle processes.
\end{abstract}

\section{Introduction}

The solar wind (SW) is a magnetized plasma mainly composed of protons and electrons that surpasses the gravitational attraction of the Sun and that reaches supersonic speeds at a few solar radii from their source. When the supersonic SW plasma encounters a magnetized or conductive obstacle, a bow shock (BS) can form ahead of them. The upstream region magnetically connected to a planetary BS defines the corresponding foreshock. Inside this region, a fraction of solar wind particles are reflected from the bow shock and interact with the incoming SW (e.g., Eastwood et al., 2005; Wilson, 2016). Seen from the SW reference frame, the reflected SW protons constitute a nonthermal population which gives rise to plasma instabilities that lead to ultralow frequency (ULF) waves (e.g., Gary, 1993; Wilson, 2016). The presence of these waves is of fundamental importance since, in this collisionless region, they constitute an important channel through which there is transfer of energy and momentum between the incoming SW and the reflected particles. The foreshock is therefore characterized by the presence of backstreaming ions as well as by the generation and propagation of electromagnetic plasma waves.

Different types of backstreaming ion velocity distributions have been identified at the Earth's foreshock: field-aligned beams (FABs), intermediate, and diffuse (Burgess et al., 2012; Eastwood et al., 2005; Gosling et al., 1978; Paschmann et al., 1981; Thomsen et al., 1985; Wilson, 2016). Downstream of the field-aligned beam region, ion distributions characterized by a gyromotion around the magnetic field, that is, a nonvanishing perpendicular bulk velocity with respect to the background magnetic field, has also been reported. These gyrating ion distributions can be nongyrotropic (i.e., gyrophase bunched) or nearly gyrotropic. Two mechanisms are considered to explain the origin of such gyrating ion distributions. The first involves large-amplitude 
waves (produced by a field-aligned beam plasma instability) that trap ions in velocity space and produce a phase-bunched distribution (Hoshino \& Terasawa, 1985; Mazelle et al., 2000; Meziane et al., 2001; Mazelle et al., 2003). The second mechanism considers that a portion of the incoming solar wind that is specularly reflected at the bow shock is responsible for these distribution functions (Gosling et al., 1982; Gurgiolo et al., 1983; Meziane et al., 2004). Even though, in general, these are two possible processes to explain the observed gyrating ion distributions, there are cases in which only one of them makes predictions in agreement with the observations. For example, Mazelle et al. (2003) presented and analyzed a case study from the Cluster lon Spectrometry experiment where gyrating ion distributions were observed in association with low-frequency quasi-monochromatic waves with large amplitudes. These distributions had pitch angles inconsistent with a specular reflection mechanism at the bow shock. Moreover, these pitch angles were consistent with the theoretical value expected if the gyrating distributions were produced by a coherent nonlinear wave-particle interaction mechanism. When the waves were present, the ion distributions appeared as gyrophase-bunched gyrating distributions, while field-aligned beams were observed just adjacent to the interval of wave occurrence. In addition, the ULF waves were near cyclotron resonance with the ion parallel beams, a particle population capable of providing the free energy giving rise to the ion/ion right-hand $(\mathrm{RH})$ instability (Gary, 1993) responsible for the wave occurrence.

In principle, Earth's foreshock is not the only planetary environment where gyrophase-bunched ions could be produced as a result of wave-particle trapping. In the case of planetary obstacles that do not have an intrinsic global magnetic field such as Venus or Mars (Acuña et al., 1998; Russell et al., 1980), the presence of an extended neutral hydrogen (H) exosphere (Chaffin et al., 2015; Chaufray et al., 2008; Nagy et al., 1990) is capable of indirectly creating the necessary conditions for wave-particle interaction processes to take place at these locations. Indeed, the interaction between the Venusian and Martian atmospheres and the SW starts several planetary radii away from each planet (upstream from each BS) where exospheric $\mathrm{H}$ atoms are ionized and picked up by the SW (e.g., Yamauchi et al., 2015). Because of different ionization processes affecting both hydrogen exospheres and the relative velocity between the $\mathrm{H}$ atoms and the SW, the proton velocity distribution function at these altitudes (seen from the SW reference frame) is composed, once again, by a core of SW particles and a nonthermal population (less dense and more energetic) associated with the presence of newborn planetary ions. Given the unstable character of this type of velocity distribution function (Brinca, 1991; Cowee et al., 2012; Gary, 1991, 1993; Mazelle \& Neubauer, 1993; Sauer \& Dubinin, 2003; Sauer et al., 2001; Tsurutani, 1991; Tsurutani et al., 1989; Wu \& Davidson, 1972; Wu \& Hartle, 1974), this system is also capable of giving rise to different ULF quasi-monochromatic electromagnetic plasma waves in these regions. Particularly, waves characterized by a frequency very close to the local proton cyclotron frequency (in the spacecraft frame) have been detected upstream from the Martian and Venusian bow shocks and studied through magnetic field measurements obtained by several spacecraft (Bertucci et al., 2013; Brain et al., 2002; Connerney, Espley, DiBraccio, et al., 2015; Delva et al., 2009; Delva, Zhang, Volwerk, Magnes, et al., 2008; Delva, Zhang, Volwerk, Vörös, et al., 2008; Delva, Mazelle, Bertucci, et al., 2011; Delva, Mazelle, \& Bertucci, 2011; Mazelle et al., 2004; Romanelli et al., 2013, 2016; Ruhunusiri et al., 2015, 2016; Russell et al., 1990; Wei \& Russell, 2006; Wei et al., 2011, 2014). These waves are nearly circularly polarized and left handed, in the spacecraft or planetary frame. Theoretical studies on these observed waves also suggest that in most cases, they are right-handed polarized in the plasma rest frame and can be excited through the ion/ion RH instability (e.g., Delva, Mazelle, \& Bertucci, 2011; Gary, 1993; Gary \& Madland, 1988; Lee, 1989). They are observed to propagate quasi-parallel to the background magnetic field (propagation angles generally around $30^{\circ}$ or smaller), can have very large amplitude $(|\delta \mathbf{B} / \mathbf{B}| \lesssim 0.5)$, and can be present in association with particle trapping in velocity space. Given that the frequency of these waves is systematically observed close to the local proton cyclotron frequency, many studies refer to them as proton cyclotron waves. However, hereafter, we make reference to them as waves with frequencies near the local proton cyclotron frequency in the spacecraft frame (WPCF), to prevent confusion with the left-handed Alfvén lon Cyclotron mode (intrinsically left handed in the plasma rest frame). Complementary theoretical studies on wave-particle interaction can be found in Gendrin (1974), Hamza et al. (2006), Matsumoto (1985), and Le Quéau and Roux (1987).

Figure 1 displays a basic representation of related processes that could be taking place around the induced magnetosphere of a planet with an extended exosphere. As shown, ring beam or field-aligned beam resonant plasma instabilities arising in the region upstream from the planetary bow shock or in the planetary foreshock can give rise to linear electromagnetic plasma waves. Based on their wave frequency and wave vector (source dependent, see section 2 of this paper), the subsequent large-amplitude nonlinear waves are capable 


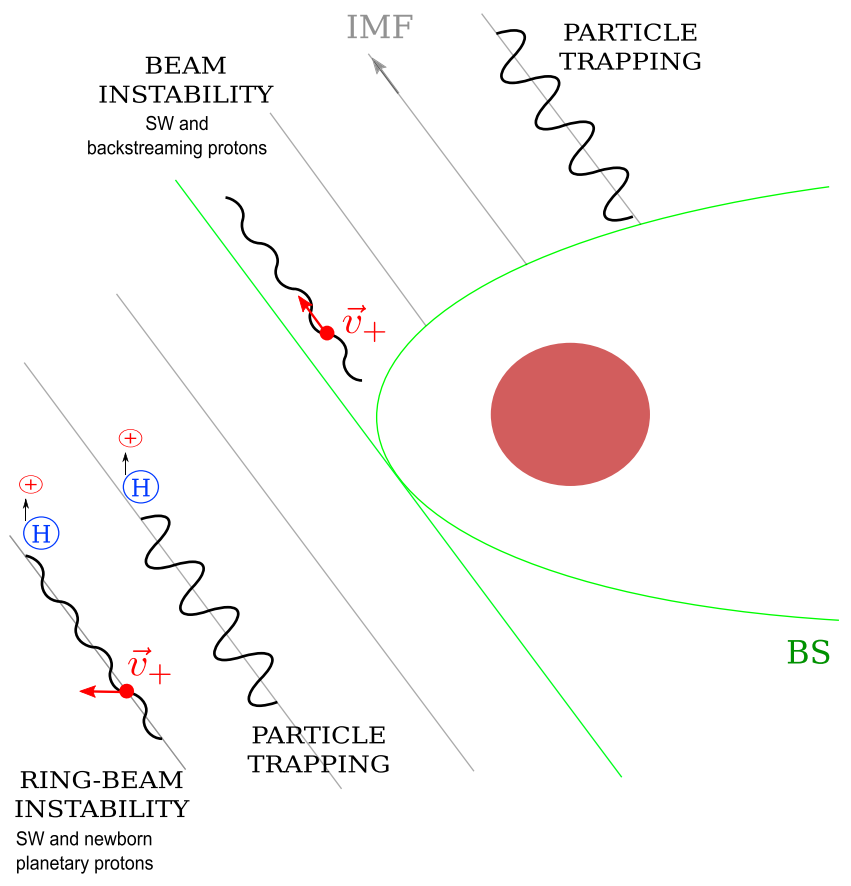

Figure 1. Schematic representation of resonant plasma instabilities taking place and defining the wave properties during the linear regime and subsequent particle trapping by a large-amplitude nonlinear wave occurring downstream from the wave generation region. Such plasma instabilities arise as a result of the interaction between SW protons and newborn or backstreaming solar wind protons (whose initial velocities are represented with red arrows). of trapping charged particles located downstream from both wave generation regions. With this in mind, the aim of the present work is to improve our current understanding of the wave-particle coupling arising in these planetary plasma environments. Particularly, one objective is to provide theoretical predictions to be tested with in situ plasma and magnetic field observations in locations where newborn planetary and backstreaming ions interact with large-amplitude waves being convected by the SW under stable conditions. For this purpose, we determine the trajectory of charged particles interacting with nonlinear parallel propagating right-handed circularly polarized waves for arbitrary initial conditions (resonant or nonresonant) and for different wave amplitudes. By considering the superposition of two circularly polarized waves with the same phase velocity, we also study the effects that wave ellipticity introduces on the charged particle's dynamics.

In this study we make use of a test particle approach. Given that the newborn planetary and backstreaming ion densities are much lower than that of the SW protons, the ion beam or ring beam components are strongly modified by the presence of large-amplitude waves, while small effects on the ion core take place. Under these conditions, the test particle approach is justified.

The present work is structured as follows. In section 2 we briefly point out the relationship between the wave properties and the suprathermal proton source velocity and gyrofrequency. In section 3, we analyze the dynamics of a charged particle forced by a large-amplitude parallel propagating right-handed circularly polarized electromagnetic wave. In particular, we display the particle's equations of motion, we derive the formal solution in velocity space, and we determine the number and type of fixed points in velocity space and their dependence on the wave properties. In section 4 we analyze in detail case studies expected in the upstream region and foreshock of Mars and Venus. Wave ellipticity effects on the particle's dynamics are studied in section 5 . A discussion on the results is provided in section 6 . Finally, we present our conclusions in section 7.

\section{Linear Theory: The Initial Resonance Condition}

In several cases, the fastest growing plasma instability that arises as a result of FABs or ring beam distributions in the upstream region of the Martian and Venusian bow shocks and at several planetary foreshocks is the ion/ion $\mathrm{RH}$ resonant. This instability is capable of giving rise to $\mathrm{RH}$ electromagnetic plasma waves (in the SW frame) for a wide range of cone angles (e.g., Brinca \& Tsurutani, 1989; Gary, 1991, 1993; Gary \& Madland, 1988). In the SW reference frame, the suprathermal nonrelativistic ions (planetary newborn or backstreaming protons) will be in resonance with a wave of frequency $w$ and wave vector $\mathbf{k}$ if

$$
w-\mathbf{k} \cdot \mathbf{V} \pm n \Omega_{0}=0 \quad n=1,2, \ldots
$$

where $w, \mathbf{k}, \mathbf{V}$, and $\Omega_{0}$ are the wave frequency, the wave vector, the mean velocity of the suprathermal proton population, and the local proton angular gyrofrequency, respectively. Thus, when resonance between a linear $\mathrm{RH}$ wave and a proton population occurs mainly for the fundamental mode $(n=1)$ (Brinca, 1991), the wave frequency in the mean suprathermal proton population's guiding center rest frame is Doppler shifted to the local proton gyrofrequency.

Hereafter, we focus in waves propagating parallel to the background magnetic field. In these cases equation (1) implies that because of the resonant character of the plasma instability, the wave frequency and parallel wavelength of the growing wave (linear phase) are determined by the suprathermal proton (source of waves) velocity distribution. Moreover, in the cases where $w \ll \Omega_{0}$ for the maximum growth rate of the $\mathrm{RH}$ mode (linear regime, see, e.g., Gary, 1993), the parallel wave vector $k_{\|}$is defined by the mean velocity parallel to $\mathbf{B}_{0}$ of this velocity distribution function, seen from the plasma (SW) rest frame: $k_{\|} \approx \Omega_{0} / V_{\|}$. Particularly, this also shows that a necessary condition for linear RH waves to be in resonance with planetary newborn 
(or backstreaming) ions is that they are costreaming, and therefore, they propagate upstream in the SW rest frame (Brinca, 1991).

\section{Dynamics of a Charged Particle Forced by a Large-Amplitude Parallel Propagating Electromagnetic Wave}

\subsection{Equations of Motion}

We study the dynamics of a test particle with charge $q(q>0)$ and mass m subject to a RH circularly polarized electromagnetic wave with frequency $w(w>0)$ and wave vector $k(k>0)$ in the SW rest frame $(S)$. We consider the case of a wave propagating parallel to the background magnetic field $\mathbf{B}_{0}$. We select a coordinate system with the $z$ axis pointing along the direction of propagation of the wave $k$, that is, $\mathbf{k}=k \hat{\mathbf{z}}$. The $\mathrm{RH}$ wave is denoted as follows:

$$
\begin{gathered}
\delta \mathbf{B}=\delta B(\sin (\sigma(k z-w t)) ; \cos (\sigma(k z-w t)) ; 0) \\
\delta \mathbf{E}=\delta E(\cos (\sigma(k z-w t)) ;-\sin (\sigma(k z-w t)) ; 0)
\end{gathered}
$$

where $\sigma= \pm 1$ for $\mathbf{B}_{0}=\sigma B_{0} \hat{\mathbf{z}}$, respectively, $\delta E=\delta B v_{\phi}$, and $v_{\phi}=w / k$ is the wave phase velocity.

Following the mathematical procedure developed in Hamza et al. (2006), we express the equations of motion in the reference frame $\left(S^{\prime}\right)$ moving at the wave phase velocity, that is $z^{\prime}=z-v_{\phi} t$. The kinetic energy of the charged particle subject to this wave is conserved only in this reference frame since there is no associated electric field. The particle gyrofrequency associated with the background magnetic field $\left|\mathbf{B}_{0}\right|$ and the wave amplitude $\delta B$ are denoted as $\Omega_{0}=q\left|\mathbf{B}_{0}\right| / m$ and $\Omega_{1}=q \delta B / m$, respectively. Given that we are concerned with nonrelativistic case studies, we approximate $\Omega_{0}^{\prime}=\Omega_{0}$ and $\Omega_{1}^{\prime}=\Omega_{1}$. Newton's equations in $S^{\prime}$ are therefore

$$
\begin{gathered}
\dot{u}_{x}=\Omega_{0} \sigma u_{y}-\Omega_{1} u_{z} \cos \left(\sigma k z^{\prime}\right) \\
\dot{u}_{y}=-\Omega_{0} \sigma u_{x}+\Omega_{1} u_{z} \sin \left(\sigma k z^{\prime}\right) \\
\dot{u}_{z}=\Omega_{1}\left[u_{x} \cos \left(\sigma k z^{\prime}\right)-u_{y} \sin \left(\sigma k z^{\prime}\right)\right]
\end{gathered}
$$

where $u_{x}, u_{y}$ and $u_{z}$ are the particle velocity components in the $S^{\prime}$ frame and

$$
\dot{z}^{\prime}=u_{z}
$$

Making a transformation to a coordinate system that takes into account the background magnetic field $\left|\mathbf{B}_{0}\right|$, we introduce (seen from $S^{\prime}$ ):

$$
\begin{gathered}
u_{\|}=\mathbf{u} \cdot \hat{\mathbf{b}}_{\mathbf{0}}=\sigma u_{z} \\
\mathbf{u}_{\perp}=\mathbf{u}-u_{\|} \hat{\mathbf{b}}_{\mathbf{0}} \\
k_{\|}=\mathbf{k} \cdot \hat{\mathbf{b}}_{0}=\sigma k
\end{gathered}
$$

with $u=\sqrt{u_{\perp}^{2}+u_{\|}^{2}}$, and the pitch angle $\alpha$ such that $\tan (\alpha)=u_{\perp} / u_{\|}$. We also normalize the variables as follows: $U=u k / \Omega_{0}, \gamma=w / \Omega_{0}, \delta=\Omega_{1} / \Omega_{0}, \tau=\Omega_{0} t$. Using these definitions, the equations describing the dynamics of a charged particle interacting with a $\mathrm{RH}$ wave propagating parallel or antiparallel to the background magnetic field direction are

$$
\begin{gathered}
\frac{\mathrm{d} U}{\mathrm{~d} \tau}=0 \\
\frac{\mathrm{d} \alpha}{\mathrm{d} \tau}=-\delta \sigma \cos \left(k_{\|} z^{\prime}+\sigma \Phi\right) \\
\frac{\mathrm{d} \Psi}{\mathrm{d} \tau}=\frac{k}{\Omega_{0}} u_{z}+\frac{\mathrm{d} \Phi}{\mathrm{d} \tau}=U \sigma \cos (\alpha)-1+\frac{\delta}{\tan (\alpha)} \sin \left(k_{\|} z^{\prime}+\sigma \Phi\right)
\end{gathered}
$$

where the polar angle in the velocity $u_{x}-u_{y}$ plane $\Phi$ is defined as $\tan (\Phi)=\sigma u_{y} / u_{x}$, and $\Psi=k z^{\prime}+\Phi$. Figure 2 displays a scheme of the circularly polarized electromagnetic wave and the wave and particle angular variables in the plane perpendicular to the background magnetic field. It is worth noticing that the phase of the magnetic field wave, measured with the same sense and origin than that for $\Phi$ is $\Phi_{B}=-k z^{\prime}+90^{\circ}$ for $\sigma=1$ and $\Phi_{B}=-k z^{\prime}+270^{\circ}$ for $\sigma=-1$. Therefore, $\Psi=\Phi-\Phi_{B}+90^{\circ}$, and $\Psi=\Phi-\Phi_{B}+270^{\circ}$, for $\sigma=1$, 

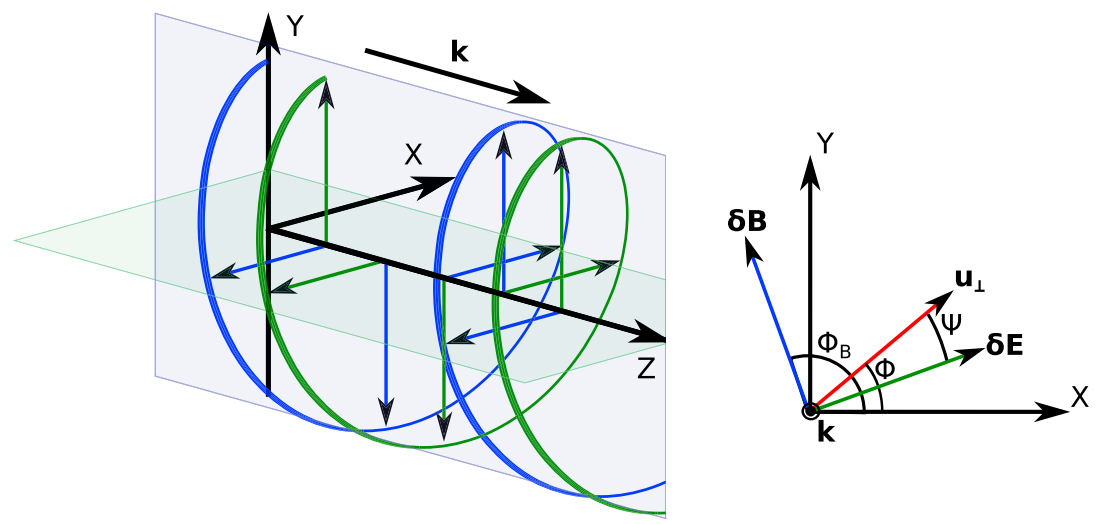

Figure 2. Circularly polarized electromagnetic plasma parallel propagating wave. (left) Three-dimensional representation where blue and green curves represent $\delta \mathbf{B}$ and $\delta \mathbf{E}$, respectively. (right) Projection of $\mathbf{u}_{\perp}$ in a plane parallel to $Z=0$, together with the local electromagnetic field and the definition of the polar angles and $\Psi$.

and $\sigma=-1$, respectively. That is, $\Psi$ basically measures the phase shift between the magnetic field wave and the component of the charge particle's velocity vector perpendicular to $\mathbf{B}_{0}$.

Equation (12) shows that a necessary condition to have a fixed point in phase space is $\left(k_{\|} z^{\prime}+\sigma \Phi\right)=90^{\circ}$ or $270^{\circ}$, so that $\frac{\mathrm{d} \alpha}{\mathrm{d} \tau}=0$. When this is the case, $\frac{\mathrm{d} \Phi}{\mathrm{d} \tau}=0$ is satisfied only if $\tan (\alpha)= \pm \delta$. To fulfill the first condition, it is therefore necessary that $u_{z}=0$. As a result, if $\delta \neq 0$, the only fixed point in phase space is $U=0$. Interestingly, there are nontrivial fixed points in velocity space around which the point representing a test particle state (in this space) can orbit. Hereafter, we simplify the wording of this sentence by referring to test particle orbits localized around a fix point in velocity space. Based on equations (11)-(13), it is possible to derive the following two constants of the particle motion:

$$
\begin{gathered}
U(\tau)=U(\tau=0) \\
g(U(\tau), \alpha(\tau), \Psi(\tau))=g(U(\tau=0), \alpha(\tau=0), \Psi(\tau=0))
\end{gathered}
$$

with

$$
g(U(\tau), \alpha(\tau), \Psi(\tau))=(\sigma U \cos (\alpha)-1)^{2}-2 \delta U \sin (\alpha) \sin (\Psi)
$$

where $g$ is the first integral associated with the motion of the charged particle under the RH wave forcing. Indeed, it can be shown that $\frac{\mathrm{d} v}{\mathrm{~d} \tau}=-\frac{\partial g}{\partial \Psi}$, and $\frac{\mathrm{d} \Psi}{\mathrm{d} \tau}=\frac{\partial g}{\partial v}$, with $v=2 U \sigma \cos (\alpha)$. Notice that under the resonance condition shown in equation (1), $U=u k / \Omega_{0}=\frac{u k}{u_{\|} k_{\|}}=\frac{u \sigma}{u_{\|}}=\sigma / \cos (\alpha)$, where if $\sigma=1$, then $0<\alpha<90^{\circ}$ and if $\sigma=-1$, then $90^{\circ}<\alpha<180^{\circ}$. In other words, once the velocity of the charged particle parallel to $\mathbf{B}_{0}$ is fixed (in order to guarantee wave-particle resonance), $U$ and $\alpha$ are not independent. Thus, $\sigma U \cos (\alpha)-1=0$ for all $\alpha$ and for all $\tau$ as long as the linear resonance condition is fulfilled. If the particle velocity is strictly aligned (in the wave frame) with $\mathbf{B}_{0}, \alpha=0$ and $U=1$. This expression diverges when $\alpha=90^{\circ}$, reflecting the fact that such a particle cannot resonate with a right-handed wave. Since we are not imposing any additional condition on the parallel velocity, these particles could be newborn planetary ions as well as ions reflected at a planetary bock shock.

\subsection{Wave-Particle Interaction: Fixed Points in Velocity Space}

Even though one can compute the position (in velocity space) for each charged particle under the influence of a nonlinear $\mathrm{RH}$ wave for all time (see Appendix A), to determine the different types of motion expected for a proton population, it is useful to compute the fixed points in velocity space for this system, for arbitrary values of energy and wave amplitude. To study such fixed points in velocity space (independently of the location on real space), we focus on equations (11)-(13). As can be seen from equation (12), fixed points in velocity space must have $\Psi^{*}=90^{\circ}$ or $\Psi^{*}=270^{\circ}$. For a fixed value of $\sigma$, these angular coordinates correspond to a parallel and antiparallel orientation between $\mathbf{u}_{\perp}$ and $\delta \mathbf{B}$. Taking into account that $U$ is a constant of motion, the fixed points also satisfy that $\alpha^{*}$ fulfills:

$$
\left[U \sigma \cos \left(\alpha^{*}\right)-1\right]+\frac{\delta \sigma}{\tan \left(\alpha^{*}\right)}( \pm 1)=0
$$


that is, $\frac{d \Psi}{d \tau}=0$, where the \pm 1 factor arises because we have imposed $\Psi^{*}=90^{\circ}$ or $\Psi^{*}=270^{\circ}$. The solutions to equation (17) are the intersections between the periodic function $\left[U \sigma \cos \left(\alpha^{*}\right)-1\right]$ and $-\frac{\delta \sigma}{\tan \left(\alpha^{*}\right)}( \pm 1)$. Given that $\alpha^{*}$ is defined between $0^{\circ}$ and $180^{\circ}$ and depending on the values for $U, \delta$, and $\sigma$, equation (17) has one or three solutions for each critical value of $\Psi^{*}$.

To determine the nature of the fix points, we develop a first-order perturbation around these point(s):

$$
\left(\begin{array}{c}
\dot{\eta} \\
\dot{\xi}
\end{array}\right)=\left(\begin{array}{cc}
\frac{\partial \dot{\alpha}}{\partial \alpha} & \frac{\partial \dot{\alpha}}{\partial \Psi} \\
\frac{\partial \dot{\Psi}}{\partial \alpha} & \frac{\partial \dot{\Psi}}{\partial \Psi}
\end{array}\right)\left(\begin{array}{l}
\eta \\
\xi
\end{array}\right)=\left(\begin{array}{cc}
0 & \delta \sigma( \pm 1) \\
-U \sigma \sin \left(\alpha^{*}\right)-\frac{\delta \sigma}{\sin ^{2}\left(\alpha^{*}\right)}( \pm 1) & 0
\end{array}\right)\left(\begin{array}{l}
\eta \\
\xi
\end{array}\right)
$$

where $\eta=\alpha-\alpha^{*}$ and $\xi=\Psi-\Psi^{*}$, and the matrix is evaluated at the corresponding fixed point. Given that the differential matrix has null trace, all fixed points are neutrally stable centers or saddle points, depending on the sign of $\Delta=\delta\left[( \pm 1) U \sin \left(\alpha^{*}\right)+\frac{\delta}{\sin ^{2}\left(\alpha^{*}\right)}\right]$. If $\Delta>0$, the fixed point is a center. If $\Delta<0$, the fixed point is a saddle.

Based on these calculations, the possible types of fixed points are the following.

1. If we consider fixed points with $\Psi^{*}=90^{\circ}$, that is, we consider cases with the (+1) factor, $\Delta>0$ for every associated $\alpha^{*}$. That is, fixed points with $\Psi^{*}=90^{\circ}$ are always centers.

2. If we consider fixed points with $\Psi^{*}=270^{\circ}$, that is, we consider cases with the (-1) factor, $\Delta>0$ if the associated $\alpha^{*}$ fulfills that $\sin \left(\alpha^{*}\right)<(\delta / U)^{1 / 3}$. In other words, the fixed points with $\Psi^{*}=270^{\circ}$ are centers when this condition is satisfied. In the opposite case, the fixed point is a saddle.

It is worth noticing that if $\alpha^{*}$ constitutes a solution to equation (17) for a fixed value of $\sigma$ and $\Psi^{*}$ (either $90^{\circ}$ or $270^{\circ}$ ), then $-\sigma$ and $180^{\circ}-\alpha^{*}$ is also a solution (with the same stability) for the same value of $\Psi^{*}$. Under the latter configuration the direction of $\mathbf{B}_{0}$ is reversed, but the pitch angle of the particle does not physically changed from the previous case (this angle is measured with respect to $\mathbf{B}_{0}$ ). As explained before, the change in the sign of $\sigma$ implies a phase shift in the definition of $\Psi$ so that if in the first case, $\mathbf{u}_{\perp}$ and $\delta \mathbf{B}$ are in phase, in the second one they are in antiphase, and vice versa. In conclusion, if the coordinates of a vector $\mathbf{u}$ constitute a stable (unstable) fixed point in velocity space for a given (local) $\mathbf{B}_{0}+\delta \mathbf{B}$, then the coordinates of $\mathbf{u}$ also constitute a stable (unstable) solution for $-\left(\mathbf{B}_{0}+\delta \mathbf{B}\right)$.

These results show that each particle interacting with the nonlinear wave under consideration will perform one of the following three possible motions in velocity space, depending on the initial conditions, the wave amplitude, and the energy of the particle: (a) The particle will be orbiting around a center, (b) The particle will be untrapped, and (c) the particle will have a fixed pitch angle and phase shift with respect to $\delta \mathbf{B}$, when the initial coordinates of the particle $(\alpha, \Psi)$ coincide with a center.

Figure 3 displays an example of the intersections between the function $\left[U \sigma \cos \left(\alpha^{*}\right)-1\right]$ (in blue) and the functions $-\frac{\delta \sigma}{\tan \left(\alpha^{*}\right)}( \pm 1)$ (in green for $\Psi^{*}=90^{\circ}$ and in red for $\Psi^{*}=270^{\circ}$ ). Both panels display the fixed points in velocity space associated with wave-particle interaction for parallel propagating waves $(\sigma=1)$ with a normalized wave amplitude $\delta=0.25$. Figure 3 (left and right) corresponds to a wave that can be originally excited by a field-aligned beam $\left(U_{0}=1\right)$ and a ring beam with a mean pitch angle of $60^{\circ}\left(U_{0}=1 / \cos \left(60^{\circ}\right)\right)$, respectively. Indeed, given that $U(\tau)=U(\tau=0)=U_{0}$, this variable allows us to determine the temporal evolution of particles (interacting with the large-amplitude wave) with the same normalized energy (in the wave frame) than the ones that are capable of giving raise to the wave (linear limit). Since $U_{0}=1 /\left|\cos \left(\alpha_{0}\right)\right|$ (linear wave-particle resonance), the lower value of $U$ associated with resonant particles giving rise to $\mathrm{RH}$ waves is $U_{0}=1$, that is, the one associated with FABs. As shown in Figure 3, the number (and type) of fixed points for the wave-particle interaction system varies depending on the value of $U$. In particular, Figure 3 (left) displays two intersection points, corresponding to two centers in velocity space whose coordinates are $\left(\alpha \sim 43^{\circ}, \Psi=90^{\circ}\right)$ and $\left(\alpha \sim 173^{\circ}, \Psi=270^{\circ}\right)$. When the value of $U_{0}$ is increased (Figure 3, right), there are four intersection points, whose coordinates in velocity space are $\left(\alpha \sim 64^{\circ}, \Psi=90^{\circ}\right),\left(\alpha \sim 15^{\circ}, \Psi=270^{\circ}\right),\left(\alpha \sim 54^{\circ}, \Psi=270^{\circ}\right)$, and $\left(\alpha \sim 175^{\circ}, \Psi=270^{\circ}\right.$ ). Among the latter three fixed points, the one with the intermediate value of $\alpha^{*}$ is the saddle. Similar changes in the number and type of fixed points of this interacting system take place when considering cases with the same value of $U$, but different values of $\delta$. In the limit of $\delta$ taking sufficiently small values, the fixed points $\alpha^{*}$ tend toward the values corresponding to the linear resonance condition, $\alpha^{*} \sim \delta /(U-1)$ and $\alpha^{*} \sim 180^{\circ}[1-\delta / \pi(U+1)]$ (see equation (17)). 

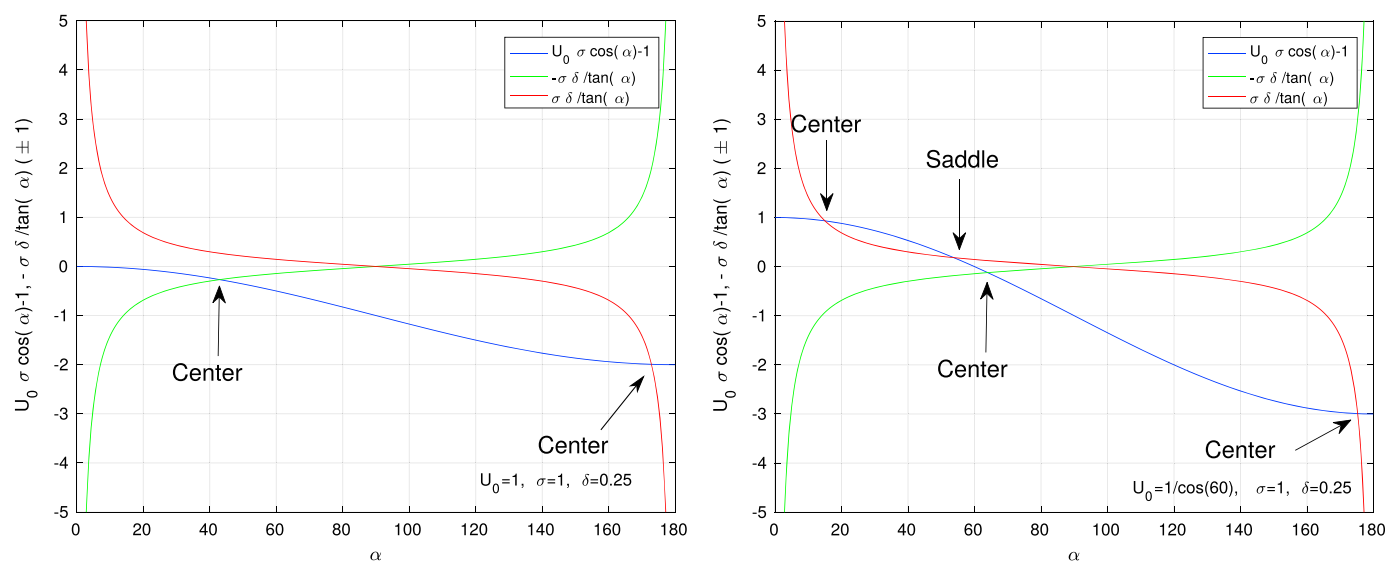

Figure 3. Pitch angle coordinate of fixed points in velocity space for the wave-particle interaction system for $\sigma=1$ and $\delta=0.25$. (left) $U_{0}=1$ and (right) $U_{0}=1 / \cos \left(60^{\circ}\right)$.

\section{Particle's Trajectory in the $\alpha-\Psi$ Velocity Plane: Main Features and Range of Validity}

4.1. Cases Study: Backstreaming Protons in Planetary Foreshocks - Newborn Planetary Protons in the Upstream Region of Mars and Venus

Figure 4 displays the variation of the $\alpha-\Psi$ velocity coordinates of charged particles (in black) interacting with a large-amplitude RH circularly polarized monochromatic electromagnetic wave for different values of $U_{0}$ and $\delta$. Figures $4 \mathrm{a}$ and $4 \mathrm{~b}, 4 \mathrm{c}$ and $4 \mathrm{~d}$, and $4 \mathrm{e}$ and $4 \mathrm{f}$ are associated with $U_{0}=1, U_{0}=1 / \cos \left(36^{\circ}\right)$, and $U_{0}=1 / \cos \left(57^{\circ}\right)$, respectively. Fixed values of $\delta=0.25$ and $\delta=0.50$ are used for Figures $4 \mathrm{a}, 4 \mathrm{c}$, and $4 \mathrm{e}$, and $4 \mathrm{~b}, 4 \mathrm{~d}$, and $4 \mathrm{f}$, respectively. All panels correspond to parallel propagating waves, that is, $\sigma=1$ and also display the separatrix (in red) between different types of trajectories. The variability of $g\left(U_{0}, \alpha, \Psi\right)$ in regions with close trajectories is significantly smaller compared to that in regions with untrapped trajectories. Thus, to make Figure 4 more understandable, we choose to show representative trajectories for each region instead of displaying uniformly separated contour levels of $g\left(U_{0}, \alpha, \Psi\right.$ ) (which would demand a large amount of curves in the untrapped region without providing new information). The blue crosses identify the fixed points (stable or unstable) in this velocity plane. Given that their horizontal coordinate is $\Psi^{*}=90^{\circ}$ or $\Psi^{*}=270^{\circ}$, we only show the corresponding value of the pitch angle.

A value of $U_{0}=1$ implies that the large-amplitude interacting waves related to Figures $4 \mathrm{a}$ and $4 \mathrm{~b}$ could have been previously excited by a field-aligned beam interacting with the SW protons through the ion-ion $\mathrm{RH}$ resonant instability. Thus, these panels are associated with particle dynamics expected to take place in planetary foreshocks (downstream from the wave generation region). As seen, there are two stable fixed points and two separatrix for both $\delta=0.25$ and $\delta=0.50$. In particular for Figure $4 \mathrm{a}, \alpha^{*}=42.97^{\circ}$ when $\mathbf{u}_{\perp}$ is in phase with $\delta \mathbf{B}$ ( $\mathbf{k}$ is parallel to $\mathbf{B}_{0}$ ) and $\alpha^{*}=172.8^{\circ}$ when $\mathbf{u}_{\perp}$ and $\delta \mathbf{B}$ are in antiphase. Therefore, this shows that waves that could have arose (linear limit) by field-aligned beams will tend to trap particles with the same energy (in the wave rest frame) around these two pitch angle centroids. Given the test particle approach, it is not possible to determine which of the two will be more populated with charged particles. However, by considering the trapping area around each of them, one might argue that the pitch angle centroid with $\alpha^{*} \sim 43^{\circ}$ is favored in this scenario. When increasing the value of $\delta$, the pitch angle centroids are shifted by $\sim 9^{\circ}$ and $7^{\circ}$, respectively. In both cases the angle between the direction of the velocity of the particle and the background magnetic field as well as the trapping areas increases.

In analogy with Figures $4 \mathrm{a}$ and $4 \mathrm{~b}$, the value of $U_{0}$ for the middle and lower ones implies that the associated large-amplitude waves could have been previously excited by a ring beam newborn planetary proton distribution interacting with the SW protons through the ion-ion $\mathrm{RH}$ resonant instability. Given the particular values of $U_{0}$, Figures $4 \mathrm{c}$ and $4 \mathrm{~d}$, and $4 \mathrm{e}$ and $4 \mathrm{f}$ are related with particle dynamics expected to take place in the upstream region of Venus and Mars, respectively. Indeed, the value of $\alpha_{0}$ is basically the interplanetary magnetic field (IMF) cone angle (Parker spiral) around these planetary environments. One must interpret these plots with caution as the initial pitch angle of the newborn planetary ions that give rise to the wave (linear limit) in the wave rest frame differs from that of in the SW frame. Very often, this difference is not large since 
Trajectory of charged particles in the $\alpha-\psi$ velocity plane
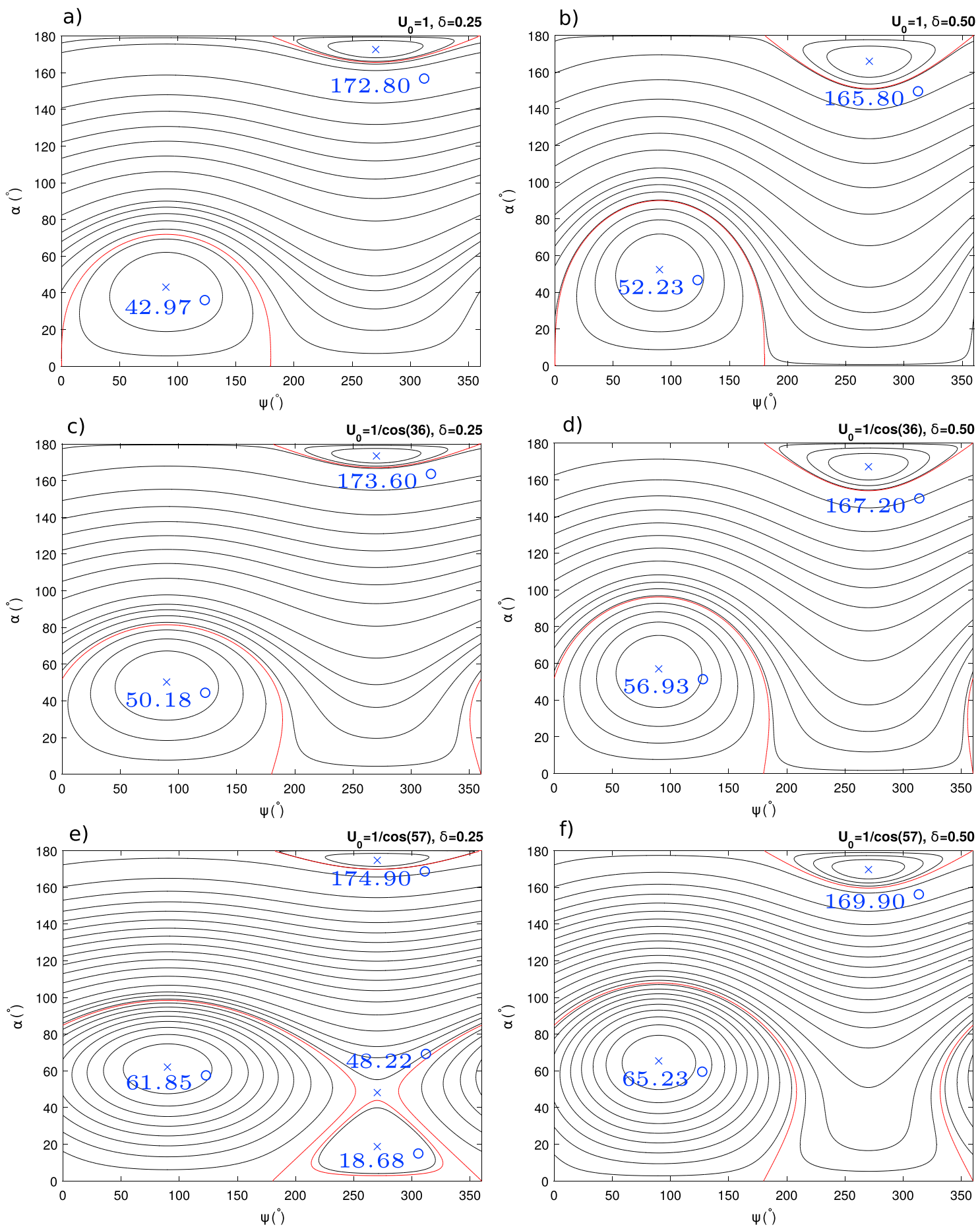

Figure 4. (a-f) Variation of the $\alpha-\Psi$ velocity coordinates of a charged particle interacting with a nonlinear RH circularly polarized monochromatic electromagnetic wave, for different values of $U_{0}$ and $\delta$ (in black). The separatrix and fixed points are displayed in red and blue, respectively. Pitch angle of the centroids are also shown in blue. 


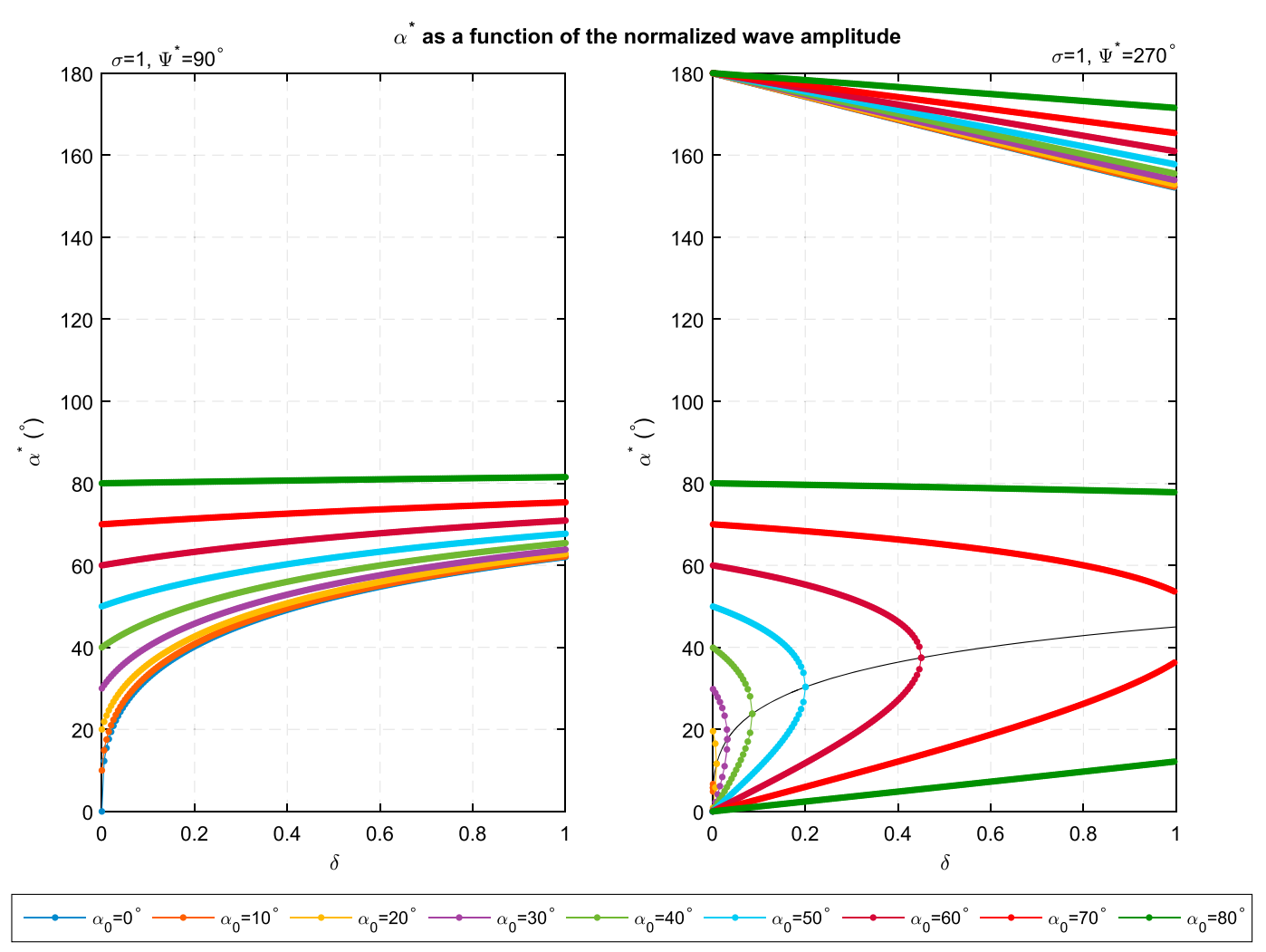

Figure 5. Pitch angle of fixed points as function of $\delta$ and color coded according to $\alpha_{0}$. (left) $\Psi^{*}=90^{\circ}$ and (right) $\Psi^{*}=270^{\circ}$.

it basically depends upon the wave phase speed, which is much smaller that the SW speed (around 1 order of magnitude smaller). In the next section we perform a parametric study to determine all the velocity fixed points with $\alpha_{0}$ varying from 0 up to $80^{\circ}$, allowing to overcome this limitation.

As can be seen in Figures $4 \mathrm{c}$ and $4 \mathrm{~d}\left(U_{0}=1 / \cos \left(36^{\circ}\right)\right)$, the main properties are similar to the cases previously analyzed. There are two stable fixed points and two separatrix for the explored values of $\delta$. Moreover, the variability of the pitch angles centroids and trapping area with the normalized amplitude resembles Figure 4a and $4 \mathrm{~b}$. When the wave amplitude is relatively small, the pitch angle centroid with largest trapping area has $\alpha^{*}=50.18^{\circ}$. For $\delta=0.50$, this centroid is located at $\alpha^{*}=56.93^{\circ}$. Figure $4 \mathrm{e}\left(U_{0}=1 / \cos \left(57^{\circ}\right)\right)$ shows a case in which four centroids are present: three of them are stable $\left(\alpha^{*}=61.85^{\circ}, \alpha^{*}=18.68^{\circ}\right.$, and $\left.\alpha^{*}=174.90^{\circ}\right)$, while the one at $\alpha^{*}=48.22^{\circ}$ is unstable. The corresponding three separatrix can also be seen. When increasing the value of $\delta$ (Figure 4f), we appreciate that two of these fixed points (with different stability) are no longer present. In other words, when the wave has a sufficiently large amplitude, we recover back a scenario closer to Figures $4 \mathrm{a}-4 \mathrm{~d}$. By comparing Figures $4 \mathrm{e}$ and $4 \mathrm{f}$, we notice that the wave amplitude determines the pitch angle coordinate of the fixed points and the number and area of the close trapping regions.

\subsection{The Trapping Centroids: Dependence on the Parameters}

Figure 5 displays the numerically determined roots to equation (17) as a function of $\delta$ and color coded by $\alpha_{0}=\operatorname{acos}\left(1 / U_{0}\right)$. Figure 5 (left and right) correspond to fixed points associated with $\Psi^{*}=90^{\circ}$ and $\Psi^{*}=270^{\circ}$, respectively. Without losing generality, this figure has been performed with $\sigma=1$. As explained in section 3 , the angle between $\mathbf{u}$ and $\mathbf{k}$ does not vary when considering $\sigma=-1$. They only difference is that the phase between $\mathbf{u}_{\perp}$ and $\delta \mathbf{B}$ is shifted by $180^{\circ}$.

As can be seen in Figure 5 (left), the value of these stable pitch angle centroids (with $\Phi-\Phi_{B}=0$ ) increases with both $\delta$ and $\alpha_{0}$. For each set of fixed points with the same value of $U_{0}$, the minimum $\alpha^{*}$ is the one that fulfills the corresponding linear resonance condition (i.e., takes place for $\delta=0$ ). Also notice that the range of values that $\alpha^{*}$ takes (with different $\delta$ ) decreases with increasing values of $\alpha_{0}$. In other words, nonlinear waves originally excited by ring beam distributions with large mean pitch angles tend to trap particles around the same value. In contrast with this, large-amplitude waves originally excited by field-aligned beams are capable 
of trapping particles around much larger pitch angles. For example, while $\alpha^{*}=52.23^{\circ}$ for $\alpha_{0}=0^{\circ}$ and $\delta=0.5$, $\alpha^{*}=80.82^{\circ}$ for $\alpha_{0}=80^{\circ}$ and the same normalized wave amplitude. As can also be seen, the rate of change of $\alpha^{*}$ with $\delta$ decreases with increasing values of $\delta$ (for a fix value of $U_{0}$ ). In addition, the rate of change of $\alpha^{*}$ with $\alpha_{0}$ increases with increasing values of $\alpha_{0}$ (for a fix value of $\delta \neq 0$ ). As a result, the range of pitch angle centroids consistent with a fixed value of $\delta$ is reduced as the normalized wave amplitude increases. All curves shown in Figure 5 (left) tend toward $90^{\circ}$ for waves with sufficiently large amplitudes.

Figure 5 (right) displays the fix points with $\Phi-\Phi_{B}=180^{\circ}$. As expected, there are one or three possible values of $\alpha^{*}$ consistent with a fix value for $\delta$ and $\alpha_{0}$. When three roots are present, the one with the intermediate value of $\alpha^{*}$ is the unstable fixed point. The remaining two are centroids. Given a value of $\alpha_{0}$, there is only one root (center) for a wave amplitude sufficiently large. For example, let us focus in the $\alpha_{0}=60^{\circ}$ curve. For a fixed $\delta$ smaller than 0.45 there are three fixed points. When reaching $\delta \sim 0.45$, two fixed points of opposite stability are merged remaining only the stable fixed point whose $\alpha^{*} \sim 171^{\circ}$. The black curve displays the value of $\alpha$ at which the merging of two fixed points takes place, as a function of $\delta$. $U_{0}$ must take a particular value that depends upon the value of $\delta$ to see such behavior. When considering the roots in the upper branch associated with a fix $\alpha_{0}$, the value of $\alpha^{*}$ decreases with increasing $\delta$. In the other limit, when $\delta=0$, the roots are $0^{\circ}, 180^{\circ}$ and the corresponding value of $\alpha_{0}$ (linear resonance). Interestingly and in contrast to Figure 5 (left), large-amplitude waves originally excited by a ring beam distribution (with large $\alpha_{0}$ ) can, in principle, trap particles around pitch angles significantly different if $\Psi^{*}=270^{\circ}$. On the other hand, both panels show that particle trapping is more difficult (or does not occur if $\Psi=90^{\circ}$ ) to take place when $\alpha>90^{\circ}$ for the $\mathrm{RH}$ mode wave discussed here. Such difference in the selected pitch angle centroids is the reflect of the impossibility for a linear RH wave to resonate with charged ions moving in the opposite direction to $\mathbf{k}$ (when $\mathbf{k} \| \mathbf{B}_{0}$ ). Notice that the number of centroids with pitch angles in this range increases as $\delta$ increases. We recall that intrinsically left-handed resonant waves can be excited by ring beam distributions with large values of $\alpha_{0}$ (Brinca \& Tsurutani, 1989) and may trap charged particles with a strong ring component. This case is outside the scope of the present study.

Finally, it is worth stressing that the fixed points in velocity space satisfy:

$$
\frac{\mathrm{d} \Phi}{\mathrm{d} \tau}=-1+\frac{\delta}{\tan \left(\alpha^{*}\right)}( \pm 1)
$$

This equation shows that the angular frequency of the particle that is interacting with a finite amplitude wave is equal to the local ion gyrofrequency plus or minus a frequency dependent on the normalized wave amplitude. This particle gryofrequency together with the pitch angle centroid constitutes two theoretical predictions (in the wave frame) to be determined when analyzing plasma measurements displaying wave-particle interaction signatures.

\section{Wave Ellipticity Effects}

As mentioned in section 1, nearly circularly polarized, large-amplitude waves have been observed in the space plasma environments of interest. However, departures from ellipticity equal to 1 have often been detected in such measurements. Motivated by this, we study the effects that wave ellipticity has on the evolution of charged particles in velocity space. We focus this analysis on charged particles with initial pitch angle and gyrophase equal to that of fixed points found for circularly polarized waves in the Martian and Venusian environments.

We represent an elliptically polarized parallel propagating wave as the superposition of two circularly polarized waves with the same phase velocity:

$$
\delta \mathbf{B}=\delta \mathbf{B}_{1}+\delta \mathbf{B}_{2}
$$

where

$$
\begin{aligned}
& \delta \mathbf{B}_{1}=\left|\delta \mathbf{B}_{1}\right|\left[\sin \left(\sigma\left(k_{1} z-w_{1} t\right)\right) ; \cos \left(\sigma\left(k_{1} z-w_{1} t\right)\right) ; 0\right] \\
& \delta \mathbf{B}_{2}=\left|\delta \mathbf{B}_{2}\right|\left[\sin \left(\sigma\left(k_{2} z-w_{2} t\right)\right) ; \cos \left(\sigma\left(k_{2} z-w_{2} t\right)\right) ; 0\right]
\end{aligned}
$$

and

$$
\frac{w_{1}}{k_{1}}=\frac{w_{2}}{k_{2}}=v_{\phi} ; k_{2}=-k_{1} ; w_{2}=-w_{1}
$$


Given that both waves have the same phase speed, we solve the problem in the reference frame where the electric field is 0 . Once again, the energy of the particle is conserved, and the remaining equations for $\Psi_{1}=k_{1} z^{\prime}+\Phi, \Psi_{2}=k_{2} z^{\prime}+\Phi$ and the particle's pitch angle are

$$
\begin{gathered}
\frac{\mathrm{d} \alpha}{\mathrm{d} \tau}=-\delta_{1} \sigma\left[\cos \left(\sigma \Psi_{1}\right)+r_{1,2} \cos \left(\sigma \Psi_{2}\right)\right] \\
\frac{\mathrm{d} \Psi_{1}}{\mathrm{~d} \tau}=\frac{k_{1}}{\Omega_{0}} u_{z}+\frac{\mathrm{d} \Phi}{\mathrm{d} \tau}=U_{1} \sigma \cos (\alpha)-1+\frac{\delta_{1}}{\tan (\alpha)}\left[\sin \left(\sigma \Psi_{1}\right)+r_{1,2} \sin \left(\sigma \Psi_{2}\right)\right] \\
\frac{\mathrm{d} \Psi_{2}}{\mathrm{~d} \tau}=\frac{k_{2}}{\Omega_{0}} u_{z}+\frac{\mathrm{d} \Phi}{\mathrm{d} \tau}=-U_{1} \sigma \cos (\alpha)-1+\frac{\delta_{1}}{\tan (\alpha)}\left[\sin \left(\sigma \Psi_{1}\right)+r_{1,2} \sin \left(\sigma \Psi_{2}\right)\right]
\end{gathered}
$$

where $U_{1}=k_{1} u / \Omega_{0}$, and $r_{1,2}=\left|\delta \mathbf{B}_{2}\right| /\left|\delta \mathbf{B}_{1}\right|$.

Notice that $\dot{\Psi}_{1}$ and $\dot{\Psi}_{2}$ cannot be simultaneously null. If this were possible, then $\dot{\Psi}_{2}-\dot{\Psi}_{1}=0$, the latter being only fulfilled if $\cos (\alpha)=0$. Such condition implies, however, $\dot{\Psi}_{1}=\dot{\Psi}_{2}=-1$, showing that both quantities cannot be constant at the same time. Thus, even $\dot{\Psi}_{1}$ and $\dot{\alpha}$ can be both 0 at time $\tau=\tau_{0}$ (by means of a combination of $\Psi_{1}, \Psi_{2}$ and $\alpha$ values), $\dot{\Psi}_{2}$ cannot be null at the same time. Under these conditions, $\Psi_{2}\left(\tau=\tau_{0}+\delta \tau\right) \neq \Psi_{2}\left(\tau=\tau_{0}\right), \Psi_{1}\left(\tau=\tau_{0}+\delta \tau\right)=\Psi_{1}\left(\tau=\tau_{0}\right)$ and $\alpha\left(\tau=\tau_{0}+\delta \tau\right)=\alpha\left(\tau=\tau_{0}\right)$. Therefore, $\dot{\Psi}_{1}$ and $\dot{\alpha}$ are not longer equal to zero at time $\tau=\tau_{0}+\delta \tau$. This shows that there is not a centroid point in the $\Psi_{1}-\alpha$ plane: if a particle reaches a location where the derivatives of $\Psi_{1}$ and $\alpha$ are 0 , a time $\delta \tau$ later, these derivatives will not be zero as a result of changes in the $\Psi_{2}$ variable. This is an interesting difference when compared to the dynamics of a particle under a strictly circularly polarized wave.

By comparing the rates of change of the three variables, the following additional points can be drawn:

1. $\dot{\alpha}$ takes values on the order of $\delta_{1}$, the normalized wave amplitude.

2. To have particle motion restricted to a closed region in the $\Psi_{1}-\alpha$ plane, it is necessary that $\dot{\Psi}_{1}$ be $\mathrm{O}\left(\delta_{1}\right)$. For particles with $\Psi_{1}$ close to $90^{\circ}$ or $270^{\circ}$ (close to be in phase or antiphase with $\delta \mathbf{B}_{1}$ ), $\left[U_{1} \sigma \cos (\alpha)-1\right]$ must be $\mathrm{O}\left(\delta_{1}\right)$ and $\tan ^{-1}(\alpha)$ must be $\mathrm{O}(1)$.

3. In general, $\dot{\Psi}_{1}$ and $\dot{\Psi}_{2}$ take extremely large values when $\alpha$ is close to $0^{\circ}$ and $180^{\circ}$, except for a very particular combination of $\Psi_{1}$ and $\Psi_{2}$ values where $\left[\sin \left(\sigma \Psi_{1}\right)+r_{1,2} \sin \left(\sigma \Psi_{2}\right)\right]=0$. The latter condition will be satisfied for a small time interval since $\dot{\Psi}_{1}$ and $\dot{\Psi}_{2}$ cannot be zero at the same time.

Despite the fact that there are not fixed points in velocity space for an elliptically polarized wave, the differences between the dynamics of particle subject to a strictly circularly polarized wave and an elliptical wave very close to being circular do not manifest abruptly. Indeed, the time interval for a particle to be displaced from a point in which $\dot{\alpha}=\dot{\Psi}_{1}=0$ depends on its location in velocity space as well as on the initial rate of change of $\Psi_{2}$. For example, Figure 6 displays the evolution of a charged particle in the $\alpha-\Psi_{1}$ velocity space when interacting with an elliptical wave with $r_{1,2}=0.033$ (in blue) and $r_{1,2}=0.066$ (in green). These results are derived by performing Runge-Kutta fourth-order (RK4) numerical integration in a time interval $\Delta t=100 T_{0}$, with $T_{0}$ being the particle gyroperiod. For each value of $r_{1,2}$, we study two cases: one with initial conditions $\Psi_{1}(0)=90^{\circ}, \Psi_{2}(0)=0^{\circ}$ and $\alpha(0)=50.18^{\circ}$. The second one has initial conditions $\Psi_{1}(0)=270^{\circ}, \Psi_{2}(0)=0^{\circ}$, and $\alpha(0)=173.60^{\circ}$. For both cases, $U_{1}=1 / \cos \left(36^{\circ}\right)$ and $\delta_{1}=0.25$. Thus, the initial conditions have the same velocity coordinates in the $\alpha-\Psi_{1}$ plane than the centroids identified in Figure 4c. For easy comparison, we have also added the dynamical evolution of particles that interact with a strictly circularly $\left(r_{1,2}=0\right)$ polarized wave (black dashed lines), keeping the remaining parameters the same. Figure 6 also displays the corresponding separatrix (red dashed lines) already shown in Figure 4. As can be seen, if the ellipticity of the wave is slightly increased $\left(r_{1,2}=0.033\right)$, the $\alpha-\Psi_{1}$ coordinates of each charge particle under study are not fixed, as is the case for $r_{1,2}=0$. Instead, they vary with time and sample a region (in blue) in the $\alpha-\Psi_{1}$ plane. Interestingly, the size of this region is significantly larger when the particle's initial condition is that of the centroid with larger pitch angle (for the circular polarization case). When the wave ellipticity is $r_{1,2}=0.066$, the range of $\alpha-\Psi_{1}$ coordinates (in green) taken by each charge particle is increased even further. This figure also shows that particles with $\Psi_{1}(0)=270^{\circ}, \Psi_{2}(0)=0^{\circ}$, and $\alpha(0)=173.60^{\circ}$ are untrapped when interacting with such elliptical wave. In contrast with this, particles with $\Psi_{1}(0)=90^{\circ}, \Psi_{2}(0)=0^{\circ}$ and $\alpha(0)=50.18^{\circ}$ still sample a narrow region centered on $\Psi_{1}=90^{\circ}$ with $\Delta \Psi_{1}=\Psi_{1}^{\max }-\Psi_{1}^{\min } \sim 0.1^{\circ}$ and with $\Delta \alpha=\alpha^{\max }-\alpha^{\min } \sim 1.25^{\circ}$. 


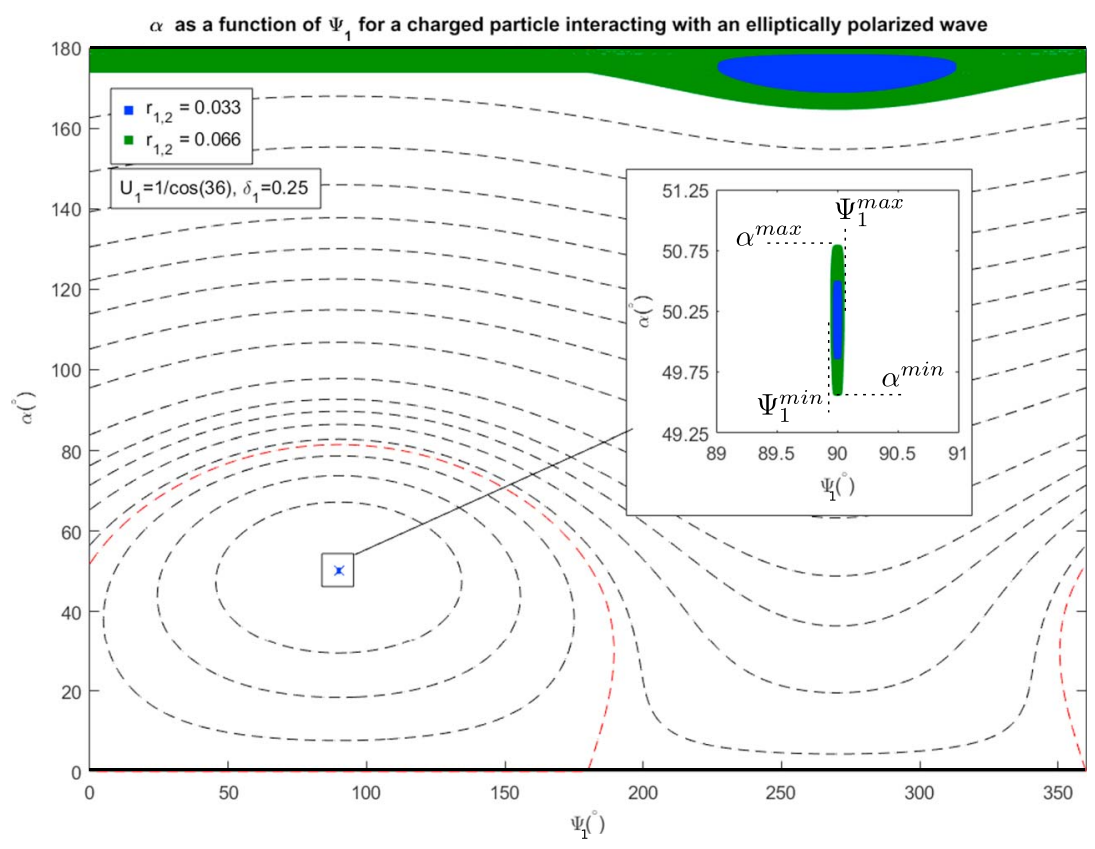

Figure 6. $\alpha$ as a function of $\Psi_{1}$ for a charged particle interacting with an elliptically polarized wave: $r_{1,2}=0.033$ (blue), $r_{1,2}=0.066$ (green). Dashed black lines correspond to the curves displayed in Figure $4 c\left(r_{1,2}=0\right.$, circular wave polarization).

Based on these considerations, Figure 7 displays $\Delta \alpha$ and $\Delta \Psi_{1}$ as a function of $r_{1,2}$ (top and bottom panels, respectively) for charged particles whose initial conditions are the velocity coordinates of several fixed points found for the strictly circularly polarized case (with the corresponding values of $U$ and $\delta$ ). Indeed, the color of each curve is associated with one of the centroids identified in Figure 4. Same as with Figure 6, we derive these results by performing RK4 numerical integration in a time interval $\Delta t=100 T_{0}$. The parameter $r_{1,2}$ is varied between 0 and 0.33 . We explore this range of values to be consistent with the range of ellipticity values found for WPCF in the upstream region of Mars (see, e.g., Table 1 in Romanelli et al., 2013), and also considered when analyzing WPCF in the upstream region of Venus (Delva et al., 2015). As can be seen in the top panel, $\Delta \alpha$ increases as $r_{1,2}$ increases for all curves. The four (three) curves with relatively small (large) slopes are associated with particles whose initial condition are one of the centroids with large trapping area (see Figure 4) and $\delta_{1}=0.25\left(\delta_{1}=0.50\right)$. Thus, the wave ellipticity and the wave amplitude determine the magnitude of the departure from what would be a centroid in the strictly circularly polarized wave case. The same trend is observed in the bottom panel: $\Delta \Psi_{1}$ increases as $r_{1,2}$ increases for all curves. Also, larger $\Delta \Psi_{1}$ is observed when the initial condition corresponds to one of the centroids (with large trapping area) found for a relatively large normalized wave amplitude. For all cases shown in this figure, the variability of the $\left(\alpha, \Psi_{1}\right)$ velocity coordinates of a charged particle interacting with an elliptically polarized wave remains bounded: the maximum $\Delta \alpha \sim 32^{\circ}$ and the maximum $\Delta \Psi_{1} \sim 48^{\circ}$. Interestingly, the charged particle remains inside the separatrix expected for the strictly circularly polarized case with the same values of $U$ and $\delta$.

In analogy with Figure 7, Figure 8 displays the $\Delta \alpha$ and $\Delta \Psi_{1}$ as a function of $r_{1,2}$ for charged particles whose initial conditions (color of each curve) are the velocity coordinates of some of the remaining centroids (large pitch angles) found for the strictly circularly polarized case (in Figure 4). Figure 8 only displays the cases where the evolution of a charged particle in the $\alpha-\Psi_{1}$ plane is bounded $\left(\Delta \Psi_{1}<180^{\circ}\right)$. Similarly to Figure 7, we find that $\Delta \alpha$ and $\Delta \Psi_{1}$ increase as $r_{1,2}$ increases. Also, in agreement with what is shown in Figure 6 , we find that the temporal evolution of such charged particles in the $\alpha-\Psi_{1}$ plane is significantly different when compared to the circularly polarized case. More specifically, particles become untrapped for very low values of $r_{1,2}$. Indeed, particles are untrapped for $r_{1,2}$ larger than $\sim 0.133$ for any of the cases under study. Moreover, particles are untrapped for $r_{1,2}$ larger than $\sim 0.033$ when considering the centroids with large pitch angle in Figures $4 a$ and $4 \mathrm{~b}$ as initial condition. In contrast with Figure 7 , significantly larger values of $\Delta \Psi_{1}$ are found compared 

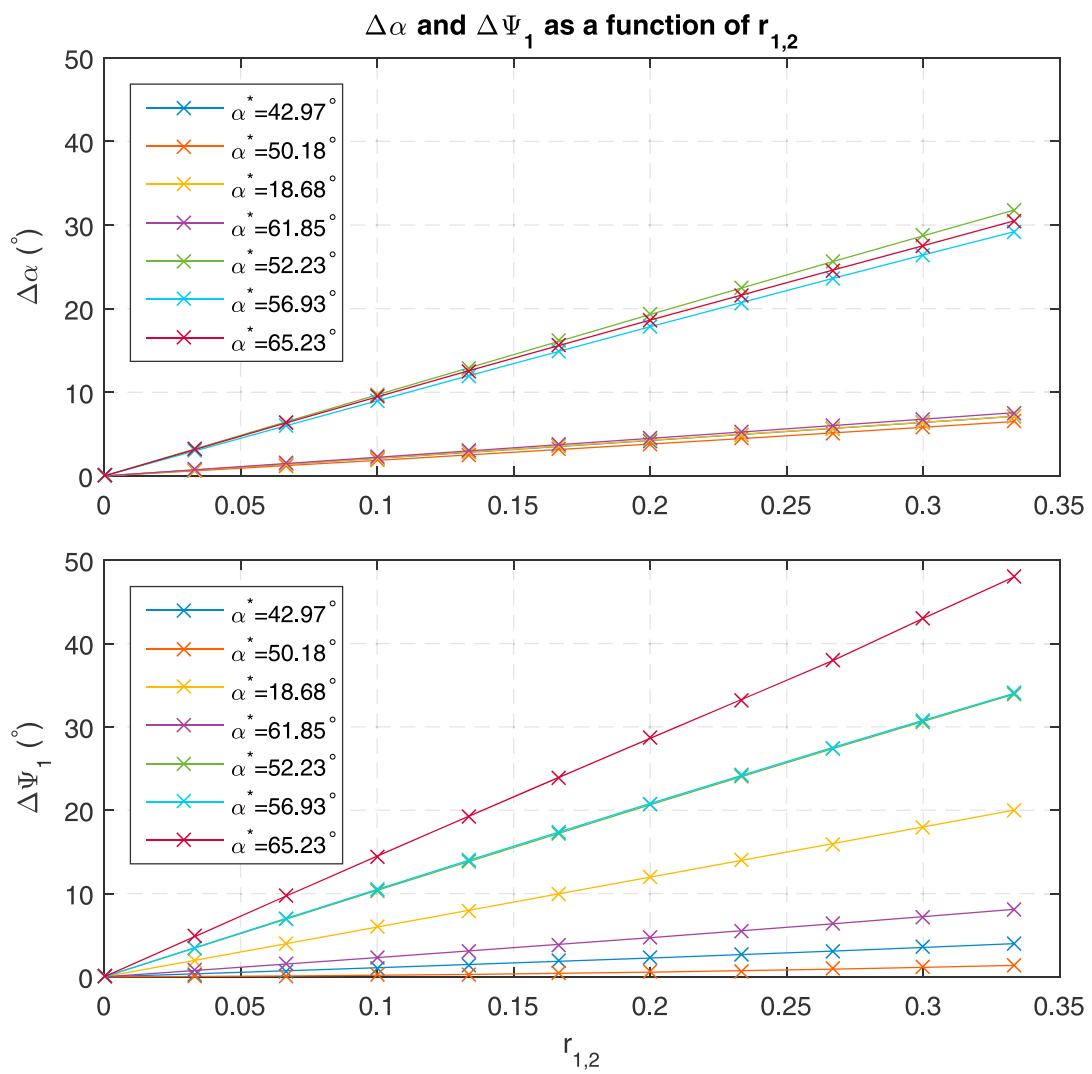

Figure 7. Ellipticity effects: $\Delta \alpha$ and $\Delta \Psi_{1}$ as a function of $r_{1,2}$ for charged particles interacting with an elliptical wave, for different initial conditions (color coded).
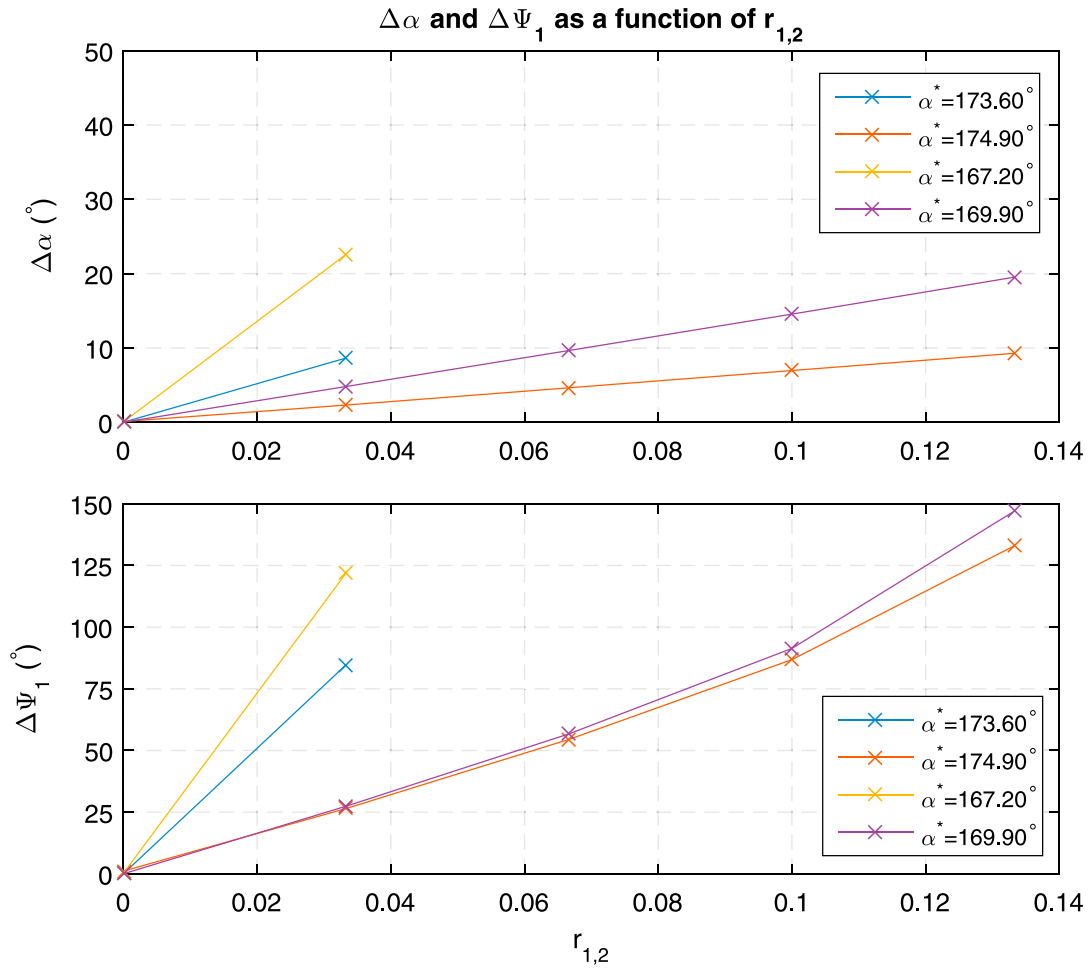

Figure 8. Ellipticity effects: $\Delta \alpha$ and $\Delta \Psi_{1}$ as a function of $r_{1,2}$ for charged particles interacting with an elliptical wave, for different initial conditions (color coded). 
to $\Delta \alpha$ for a fixed value of $r_{1,2}$. For example $\Delta \Psi_{1} \sim 147^{\circ}$ and $\Delta \alpha \sim 20^{\circ}$ when $r_{1,2}=0.1333$ and $\alpha^{*}=169.9^{\circ}$. This feature resembles the shape of the trapping area associated with the centroids with large pitch angle found for the strictly circularly polarized wave case.

\section{Discussion}

The first main goal of this work is the study of the interaction between a large-amplitude parallel propagating RH circularly polarized wave and a test charged particle from first principles, and for different initial conditions. We have computed the fixed points in velocity space associated with this interacting system. By considering different values for the waves properties and the background magnetic field, we find that only a few (or one) combinations of $\mathbf{u}_{\|}, \mathbf{u}_{\perp}$ make the total electromagnetic force to conserve the particle's energy, pitch angle, and gyrophase (respect to $\delta \mathbf{B}$ ) for all time $t$, in the wave frame. In general, each test particle is moving along the direction of propagation of the wave for all time $t$, even though their velocity coordinates might coincide with the ones of a fixed point. That is, the interacting system under study does not have a nontrivial fixed point in the phase space. In addition, we determine the stability of each of the velocity fixed points and the relationship between their coordinates in velocity space and the wave properties $(\sigma, \delta$, and $\Psi)$.

Second, making use of these theoretical calculations, we provide predictions for nominal conditions in the regions upstream from the Martian and Venusian bow shock and planetary foreshocks. As shown in Figure 4, we find that nonlinear WPCF that could have initially arise from the implantation of newborn protons from the Venusian and Martian exospheres in the SW (through the ion/ion RH resonant instability) can typically trap particles around pitch angles close to $50^{\circ}$ and $62^{\circ}$ (with the same energy in the wave frame), respectively. These are the centroids with largest trapping area associated with a Parker Spiral IMF at the Venus and Martian heliocentric distances and $\delta=0.25$. When the normalized wave amplitude is $\delta=0.50$, these pitch angle centroids are slightly shifted to $\sim 57^{\circ}$ and $\sim 65^{\circ}$, respectively. Since the cone angle can largely vary between $0^{\circ}$ and $180^{\circ}$, the theoretical model presented in this study can provide the expected value for the trapping of newborn ions for any initial pitch angle.

It is worth noticing that the conservation of $U$ for a test particle under the influence of a $\mathrm{RH}$ circularly polarized wave (in the wave frame) does not demand that the trapped particles and the source of the ion/ion $\mathrm{RH}$ instability have the same energy. In this work we have focused on this case taking into account that observations of gyrophased bunched ion velocity distribution functions at the Earth's foreshock have been explained in terms of wave-particle interaction processes, using this condition (Mazelle et al., 2000, 2003). Indeed, Mazelle et al. (2003) observed a change in the type of distribution function (from FABs to gyrophase bunched) coincident with the appearance of large-amplitude ULF waves. The highly nongyrotropic proton velocity distribution functions had large pitch angles $\left(\alpha \sim 60^{\circ}\right)$ and were inconsistent with a specular reflection mechanism at the terrestrial bow shock (Mazelle et al., 2003). This value was attributed to trapping (in velocity space) of particles with the same energy (in the wave frame) that the ones that originally gave rise to the wave from the ion/ion RH instability. In the present study we also determine the pitch angle centroids values as a function of $\delta$ and $U_{0}$ (see Figure 5), and, in particular, our results are in agreement with the case studies in Mazelle et al. $(2000,2003)$.

It is also important to emphasize that the test particle approach does not take into account the back reaction of the suprathermal population on the wave. As pointed out in section 1, this formalism is justified for time intervals where the wave amplitude is approximately constant and the nonlinear wave affects the suprathermal population dynamics without being modified significantly. In the upstream regions of Venus and Mars, WPCF have been observed to have approximately constant (and large) amplitude during time intervals on the order of 10 min or larger (e.g., Delva et al., 2015; Mazelle et al., 2004; Romanelli et al., 2013). In addition, the employed theoretical model considers strictly monochromatic waves. In this sense, although WPCF are observed to have a relatively narrow frequency peak (e.g., Delva et al., 2015; Mazelle et al., 2004; Romanelli et al., 2013, 2016) when compared to several ion foreshock modes, effects due to departures from this hypothesis must be evaluated in each particular case under study.

Finally, we have analyzed the effects that wave ellipticity has on the dynamical evolution of the charged particles. Our results show that in contrast with the circularly polarized case, there are not fixed points in velocity space. Indeed, the velocity coordinates of a charged particle whose initial conditions are equal to that of a fixed point (for a circularly polarized wave) vary with time. Because of this variability, these coordinates sample a region in the $\alpha-\Psi_{1}$ velocity plane that is found to increase with $r_{1,2}$ and $\delta$. Interestingly, charged particles 
are untrapped more easily (lower values of $r_{1,2}$ ) if the initial velocity coordinates are that of a fixed point (for the circular case) with a large pitch angle centroid ( $\mathbf{u}_{\|}$antiparallel to $\mathbf{k}$ ). In this regard, this result is consistent and provides an explanation for the observations of suptrathermal particles having pitch angle values very close to only one of the two pitch angle centroids theoretically expected for the strictly circularly polarized wave case (Mazelle et al., 2000, 2003).

Future theoretical studies could be focused on the expected signatures in velocity space for charged particles interacting with nonlinear waves propagating with small angles with respect to the background magnetic field. Indeed, as stated in section 1, observations at the terrestrial foreshock and around Mars and Venus have shown waves with small but oblique propagation. These studies would then benefit from the approach and results presented in Hamza et al. (2006) and in this work.

\section{Conclusions}

The proton velocity distribution function upstream from the Martian and Venusian bow shocks and in planetary foreshocks is composed by a core of SW particles and a secondary population of newborn planetary and/or backstreaming ions. Plasma instabilities and the subsequent wave-particle coupling constitute a stage in the dynamical evolution of these highly unstable distribution functions toward stable ones. In other words, wave-particle interaction is an important phenomena that takes place at these plasma environments as part of the thermalization of the suprathermal ion population.

In this work we study the interaction between a large-amplitude monochromatic electromagnetic righthanded plasma wave propagating parallel to the ambient magnetic field and test charged particles for different initial conditions, wave properties, and background magnetic field. Based on these analyses, we provide theoretical predictions for the expected pitch angle trapping centroids and particle gyrofrequencies in the upstream region of Mars and Venus and planetary foreshocks, under nominal conditions. These calculations are particularly useful to interpret measurements currently being provided by the Mars Atmosphere and Volatile EvolutioN (MAVEN) mission (Jakosky et al., 2015) Solar Wind Ion Analyzer (Halekas et al., 2015), and the MAVEN Magnetometer (Connerney, Espley, Lawton, et al., 2015) during time intervals with largeamplitude quasi-monochromatic WPCF. Given the substantial spatial coverage of the region upstream of the Martian bow shock, MAVEN also provides an excellent opportunity to perform planetary foreshock studies (e.g., Meziane et al., 2017). Indeed, the present study provides a mean to evaluate if observed nonthermal proton velocity distribution functions at these locations display signatures that can be understood in terms of nonlinear wave-particle processes. Finally, this work together with the methodology presented in Hamza et al. (2006) can provide the basis for future theoretical analyses that take into account both the ellipticity and the obliquity of the observed ultralow frequency waves.

\section{Appendix A: Exact time-Dependent Solution in Velocity Space-Analytical Approach for an Arbitrary Initial Condition}

Making use of the conservation of $g(\tau)$ and $U(\tau)$ and equations (12) and (13), we obtain the second-order differential equation for the charged particle's pitch angle:

$$
\frac{d^{2} \alpha}{\mathrm{d} \tau^{2}}=\sigma\left[\frac{(\sigma U \cos (\alpha)-1)^{2}-g(\tau=0)}{2 U \sin (\alpha)}\right](\sigma U \cos (\alpha)-1)+\left[\frac{(\sigma U \cos (\alpha)-1)^{2}-g(\tau=0)}{2 U \sin (\alpha)}\right]^{2} \frac{1}{\tan (\alpha)}
$$

Equation (A1) can be simplified making a change of variables in which $X$ is defined as $X=\sigma U \cos (\alpha)-1$. Notice that $X$ is equal to $-\left(w_{\text {ion }}+\Omega_{0}\right) / \Omega_{0}$, that is, it measures the closeness to the wave-particle linear resonance condition, normalized by the local ion gyrofrequency. Using once again that $g(\tau)$ is constant, we obtain the following forced Duffing equation:

$$
\frac{d^{2} X}{\mathrm{~d} \tau^{2}}=-\frac{X^{3}}{2}-X \delta^{2}\left(1-\frac{g(\tau=0)}{2 \delta^{2}}\right)-\delta^{2}
$$

where the factor $\left(1-\frac{g(\tau=0)}{2 \delta^{2}}\right)$ is the result of the coupling between equations (12) and (13). Indeed, to obtain the ordinary differential equation for $\alpha$, we have derived $\Psi$ as a function of $\alpha$ making use of the conservation of $g(\tau)=g(0)$. For a given particle, $g(\tau=0)$ is fixed based on its initial condition $(\alpha(0), \Psi(0)$ and $U(0))$. Thus, this factor is explicitly showing that the evolution of $\alpha(\tau)$ depends not only on $\alpha(0)$ but also on $\Psi(0)$, 
pointing out a rich dynamical behavior. Because we have derived the second-order ordinary differential equation (equation (A2)) making use of the conservation of $g$, the integration is valid for any initial condition under consideration (whether the particle is initially resonant with the wave or not) as long as $g(\tau=0)$ is determined consistently.

Integration of equation (A2) with respect to time explicitly shows that in the wave reference frame, the following equality holds:

$$
\frac{1}{2}\left(\frac{\mathrm{d} X}{\mathrm{~d} \tau}\right)^{2}+V_{r-b}(X, g(0), \delta)=\frac{1}{2}\left(\frac{\mathrm{d} X}{\mathrm{~d} \tau}\right)^{2}(0)+V_{r-b}(X(0), g(0), \delta) \equiv E_{o}
$$

where

$$
V_{r-b}(X, g(0), \delta)=\frac{1}{2}\left[\frac{X^{4}}{4}+\delta^{2}\left((X+1)^{2}-1-\frac{g(0) X^{2}}{2 \delta^{2}}\right)\right]
$$

and $E_{0}$ is a constant of motion of the particle. Finally, from equation (A3) we obtain that

$$
\int_{X(0)}^{X(\tau)} \frac{\mathrm{d} X^{\prime}}{ \pm \sqrt{2\left(E_{o}-V_{r-b}\left(X^{\prime}, \delta, g(0)\right)\right)}}=\int_{0}^{\tau} \mathrm{d} \tau^{\prime}
$$

Based on equation (A5), $X(\tau)$ and therefore $\alpha(\tau)$ and $\Psi(\tau)$ can in principle be derived. As can be seen, $X(\tau)$ depends on $X(0), \dot{X}(0), g(0)$, and $\delta$. This dependence is once again showing that $\alpha(\tau)$ depends on the initial condition $\alpha(0)$ and also on $\Psi(0)$.

Acknowledgments

N. R. is supported by a CDD contract from Laboratoires d'excellence Exploration Spatiale des Environnements Planétaires (LABEX-ESEP ANR 2011-LABX-030). N. R. is also indebted to the French Space Agency CNES for its support. N. R. would like to thank R. Modolo and F. Leblanc for useful discussions and suggestions. The present manuscript is theoretical, and there are no additional data to acknowledge.

\section{References}

Acuña, M. H., Connerney, J. E. P., Wasilewski, P., Lin, R. P., Anderson, K. A., Carlson, C. W., ... Ness, N. F. (1998). Magnetic field and plasma observations at Mars: Initial results of the Mars Global Surveyor Mission. Science, 279(5357), 1676-1680. https://doi.org/10.1126/science.279.5357.1676

Bertucci, C., Romanelli, N., Chaufray, J. Y., Gomez, D., Mazelle, C., Delva, M., .. Brain D. A. (2013). Temporal variability of waves at the proton cyclotron frequency upstream from Mars: Implication for Mars distant hydrogen exosphere. Geophysical Research Letters, 40, 3809-3813. https://doi.org/10.1002/grl.50709

Brain, A., Bagenal, F., Acuña, M. H., Connerney, J. E. P., Crider, D. H., Mazelle, C., ... Ness, N. F. (2002). Observations of low-frequency electromagnetic plasma waves upstream from the Martian shock. Journal of Geophysical Research, 107(A6), 1076. https://doi.org/10.1029/2000JA000416

Brinca, A. (1991). Cometary linear instabilities: From profusion to perspective, Cometary plasma processes geophysical monograph (Vol. 61, pp. 211-221). Washington, DC: American Geophysical Union.

Brinca, A., \& Tsurutani, B. T. (1989). Influence of multiple ion species on low-frequency electromagnetic wave instabilities. Journal of Geophysical Research, 94, 13,565-13,569.

Burgess, D., Möbius, E., \& Scholer, M. (2012). Ion acceleration at the Earth's bow shock. Space Science Reviews, $173,5-47$. https://doi.org/10.1007/s11214-012-9901-5

Chaffin, M. S., Chaufray, J. Y., Deighan, J., Schneider, N. M., McClintock, W. E., Stewart, A. I. F., ... Jakosky, B. M. (2015). Three-dimensional structure in the Mars H corona revealed by IUVS on MAVEN. Geophysical Research Letters, 42, 9001-9008. https://doi.org/10.1002/2015GL065287

Chaufray, J. Y., Bertaux, J. L., Leblanc, F., \& Quémerais, E. (2008). Observation of the hydrogen corona with SPICAM on Mars Express. Icarus, 195(2), 598-613.

Connerney, J. E. P., Espley, J., Lawton, P., Murphy, S., Odom, J., Oliversen, R., \& Sheppard, D. (2015). The MAVEN magnetic field investigation. Space Science Reviews, 195(1-4), 257-291. https://doi.org/10.1007/s11214-015-0169-4

Connerney, J. E. P., Espley, J. R., DiBraccio, G. A., Gruesbeck, J. R., Oliversen, R. J., Mitchell, D. L., ... Jakosky, B. M. (2015). First results of the MAVEN magnetic field investigation. Geophysical Research Letters, 42, 8819-8827. https://doi.org/10.1002/2015GL065366

Cowee, M. M., Gary, S. P., \& We, H. Y. (2012). Pickup ions and ion cyclotron wave amplitudes upstream of Mars: First results from the 1D hybrid simulation. Geophysical Research Letters, 39, L08104. https://doi.org/10.1029/2012GL051313

Delva, M., Bertucci, C., Volwerk, M., Lundin, R., Mazelle, C., \& Romanelli, N. (2015). Upstream proton cyclotron waves at Venus near solar maximum. Journal of Geophysical Research: Space Physics, 120, 344-354. https://doi.org/10.1002/2014JA020318

Delva, M., Volwerk, M., Mazelle, C., Chaufray, J.-Y., Bertaux, J.-L., Zhang, T. L., \& Vörös, Z. (2009). Hydrogen in the extended Venus exosphere. Journal of Geophysical Research, 36, L01203. https://doi.org/10.1029/2008GL036164

Delva, M., Mazelle, C., \& Bertucci, C. (2011). Upstream ion cyclotron waves at Venus and Mars. Space Science Reviews, 162(1-4), 5-24.

Delva, M., Mazelle, C., Bertucci, C., Volwerk, M., Vörös, Z., \& Zhang, T. L. (2011). Proton cyclotron wave generation mechanisms upstream of Venus. Journal of Geophysical Research, 116, A02318. https://doi.org/10.1029/2010JA015826

Delva, M., Zhang, T. L., Volwerk, M., Magnes, W., Russell, C. T., \& Wei, H. Y. (2008). First upstream proton cyclotron wave observations at Venus. Geophysical Research Letters, 35, L03105. https://doi.org/10.1029/2007GL032594

Delva, M., Zhang, T. L., Volwerk, M., Vörös, Z., \& Pope, S. A. (2008). Proton cyclotron waves in the solar wind at Venus. Journal of Geophysical Research, 113, E00B06. https://doi.org/10.1029/2008JE003148

Eastwood, J. P., Lucek, E. A., Mazelle, C., Meziane, K., Narita, Y., Pickett, J., \& Treumann, R. A. (2005). The foreshock. Space Science Reviews, 118 41-94.

Gary, S. P. (1991). Electromagnetic ion/ion instabilities and their consequences in space plasmas: A review. Space Science Reviews, 56, 373-415.

Gary, S. P. (1993). Theory of space plasma microinstabilities, Cambridge atmospheric and space series. Cambridge, UK: Cambridge University Press. 
Gary, S. P., \& Madland, C. D. (1988). Electromagnetic ion instabilities in a cometary environment. Journal of Geophysical Research, 93, $235-241$.

Gendrin, R. (1974). Phase-bunching and other non-linear processes occurring in gyroresonant wave-particle interactions. Astrophysics and Space Science, 28, 245-266.

Gosling, J. T., Asbridge, J. R., Bame, S. J., Paschmann, G., \& Sckopke, N. (1978). Observations of two distinct populations of bow shock ions in the upstream solar wind. Geophysical Research Letters, 5, 957-960.

Gosling, J. T., Thomsen, M. F., Bame, S. J., Feldman, W. C., Paschmann, G., \& Sckopke, N. (1982). Evidence for speculary reflected ions upstream from the quasi-parallel bow shock. Geophysical Research Letters, 87, 1333-1336.

Gurgiolo, C., Parks, G. K., \& Mauk, B. H. (1983). Upstream gyrophase bunched ions: A mechanism for creation at bow shock and the growth of velocity space structure through gyrophase mixing. Journal of Geophysical Research, 88, 9093-9100.

Halekas, J. S., Taylor, E. R., Dalton, G., Johnson, G., Curtis, D. W., McFadden, J. P., ... Jakosky, B. M. (2015). The Solar Wind lon Analyzer for MAVEN. Space Science Reviews, 195(1-4), 125-151. https://doi.org/10.1007/s11214-013-0029-z

Hamza, A. M., Meziane, K., \& Mazelle, C. (2006). Oblique propagation and nonlinear wave particle processes. Journal of Geophysical Research, 111, A04104. https://doi.org/10.1029/2005JA011410

Hoshino, M., \& Terasawa, T. (1985). Numerical study of the upstream wave excitation mechanism: Nonlinear phase bunching of beam ions. Journal of Geophysical Research, 90(1), 57-64.

Jakosky, B. M., Lin, R. P., Grebowsky, J. M., Luhmann, J. G., Mitchell, D. F., Beutelschies, G., ... Zurek, R. (2015). The Mars Atmosphere and Volatile Evolution (MAVEN) mission. Space Science Reviews, 195(1-4), 3-48. https://doi.org/10.1007/s11214-015-0139-x

Matsumoto, H. (1985). Coherent nonlinear effects on electromagnetic wave-particle interactions. Space Science Reviews, 42, 429-448.

Mazelle, C., Le Quéau, D., \& Meziane, K. (2000). Nonlinear wave-particle interaction upstream from the Earth's bow shock. Nonlinear Processes in Geophysics, 77, 185-190.

Mazelle, C., Meziane, K., LeQueau, D., Wilber, M., Eastwood, J. P., Reme, H., .. Balogh, A. (2003). Production of gyrating ions from nonlinear wave-particle interaction upstream from the Earth's bow shock: A case study from Cluster-CIS. Planetary and Space Science, 51, 785-795.

Mazelle, C., \& Neubauer, F. M. (1993). Discrete wave packets at the proton cyclotron frequency at Comet P/Halley. Geophysical Research Letters, 20, 153-156.

Mazelle, C., Winterhalter, D., Sauer, K., Trotignon, J. G., Acuña, M. H., Baumgärtel, K., ... Slavin, J. (2004). Bow shock and upstream phenomena at Mars. Space Science Review, 111, 115-181.

Meziane, K., Mazelle, C., Lin, R. P., LeQuéau, D., Larson, D. E., Parks, G. K., \& Lepping, R. P. (2001). Three-dimensional observations of gyrating ion distributions far upstream from the Earth's bow shock and their association with low-frequency waves. Journal of Geophysical Research, 106(A4), 5731-5742.

Meziane, K., Mazelle, C., Wilber, M., Le Quéau, D., Eastwood, J., Rème, H., .. Balogh, A. (2004). Bow shock specularly reflected ions in the presence of low-frequency electromagnetic waves: A case study. Annales Geophysicae, 22, 2325-2335.

Meziane, K., Mazelle, C. X., Romanelli, N., Mitchell, D. L., Espley, J. R., Connerney, J. E. P., . . Jakosky, B. M. (2017). Martian electron foreshock from MAVEN observations. Journal of Geophysical Research: Space Physics, 122, 1531-1541. https://doi.org/10.1002/2016JA023282

Nagy, A. F., Kim, J., \& Cravens, T. E. (1990). Hot hydrogen and oxygen atoms in the upper atmospheres of Venus and Mars. Annales Geophysicae, 8, 251-256.

Lee, M. A. (1989). Ultra-low frequency waves at comets. In B. T. Tsurutani \& H. Oya (Eds.), Plasma waves and instabilities at comets and in magnetospheres, Geophysical Monograph Series (Vol. 53, pp. 13-30). Washington, DC: American Geophysical Union.

Le Quéau, D., \& Roux, A. (1987). Quasi-monochromatic wave-particle interactions in magnetospheric plasmas. Solar Physics, 111, 59-80.

Paschmann, G., Sckopke, N., Papamastorakis, I., Asbridge, J. R., Bame, S. J., \& Gosling, J. T. (1981). Characteristics of reflected and diffuse ions upstream from the Earth's bow shock. Journal of Geophysical Research, 86, 4355-4364.

Romanelli, N., Bertucci, C., Gomez, D., Mazelle, C., \& Delva, M. (2013). Proton cyclotron waves upstream from Mars: Observations from Mars Global Surveyor. Planetary and Space Science, 76, 1-9.

Romanelli, N., Mazelle, C. X., Chaufray, J. Y., Meziane, K., Shan, L., Ruhunusiri, S., ... Jakosky, B. M. (2016). Proton cyclotron waves occurrence rate upstream from Mars observed by MAVEN: Associated variability of the Martian upper atmosphere. Journal of Geophysical Research: Space Physics, 121, 11,113-11,128. https://doi.org/10.1002/2016JA023270

Ruhunusiri, S., Halekas, J. S., Connerney, J. E. P., Espley, J. R., McFadden, J. P., Larson, D. E., ... Jakosky, B. M. (2015). Low-frequency waves in the Martian magnetosphere and their response to upstream solar wind driving conditions. Geophysical Research Letters, 42, 8917-8924. https://doi.org/10.1002/2015GL064968

Ruhunusiri, S., Halekas, J. S., Connerney, J. E. P., Espley, J. R., McFadden, J. P., Mazelle, C., ... Jakosky, B. M. (2016). MAVEN observation of an obliquely propagating low-frequency wave upstream of Mars. Journal of Geophysical Research: Space Physics, 121, 2374-2389. https://doi.org/10.1002/2015JA022306

Russell, C. T., Elphic, R. C., \& Slavin, J. A. (1980). Limits on the possible intrinsic magnetic field of Venus. Journal of Geophysical Research, 85(December), 8319-8332.

Russell, C. T., Luhmann, J. G., Schwingenschuh, K., Riedler, W., \& Yeroshenko, Ye. (1990). Upstream waves at Mars: PHOBOS observations. Geophysical Research Letters, 17(6), 897-900.

Sauer, K., \& Dubinin, E. (2003). Oscillitons and gyrating ions in a beam-plasma system. Geophysical Research Letters, $30(23), 2192$. https://doi.org/10.1029/2003GL018266

Sauer, K., Dubinin, E., \& McKenzie, J. F. (2001). New type of soliton in Bi-ion plasmas and possible implications. Geophysical Research Letters, 28, 3589-3592. https://doi.org/10.1029/2001GL013047

Thomsen, M. F., Gosling, J. T., Bame, S. J., \& Russell, C. T. (1985). Gyrating ions and large-amplitude monochromatic MHD waves upstream of the Earth's bow shock. Journal of Geophysical Research, 90, 267-273.

Tsurutani, B. T. (1991). Comets: A laboratory for plasma waves and instabilities. In Cometary plasma processes, Geophysical Monograph Series (Vol. 61, pp. 189-209). Washington, DC: American Geophysical Union.

Tsurutani, B. T., Brinca, A. L., Buti, B., Smith, E. J., Thorne, R. M., \& Matsumoto, H. (1989). Magnetic pulses with durations near the local proton cyclotron period: Comet giacobini-zinner. Journal of Geophysical Research, 94, 29-35.

Wei, H., \& Russell, C. T. (2006). Proton cyclotron waves at Mars: Exosphere structure and evidence for a fast neutral disk. Journal of Geophysical Research, 33, L23103. https://doi.org/10.1029/2006GL026244

Wei, H. Y., Cowee, M. M., Russell, C. T., \& Leinweber, H. K. (2014). lon cyclotron waves at Mars: Occurrence and wave properties. Journal of Geophysical Research: Space Physics, 119, 5244-5258. https://doi.org/10.1002/2014JA020067

Wei, H. Y., Russell, C. T., Zhang, T. L., \& Blanco-Cano, X. (2011). Comparative study of ion cyclotron waves at Mars, Venus and Earth. Planetary and Space Science, 59, 1039-1047. 
Wilson, L. B. (2016). Low frequency waves at and upstream of collisionless shocks. In Low-frequency waves in space plasmas, Geophysical Monograph Series (Vol. 216, pp. 269-291). Hoboken, NJ: John Wiley. https://doi.org/10.1002/9781119055006.ch16

Wu, C. S., \& Davidson, R. C. (1972). Electromagnetic instabilities produced by neutral-particle ionization in interplanetary space. Journal of Geophysical Research, 77(28), 5399-5406. https://doi.org/10.1029/JA077i028p05399

Wu, C. S., \& Hartle, R. E. (1974). Further remarks on plasma instabilities produced by ions born in the solar wind. Journal of Geophysical Research, 79, 283-285

Yamauchi, M., Hara, T., Lundin, R., Dubinin, E., Fedorov, A., Sauvaud, J.-A., ... Barabash, S. (2015). Seasonal variation of Martian pick-up ions: Evidence of breathing exosphere. Planetary and Space Science, 119, 54-61. https://doi.org/10.1016/j.pss.2015.09.013 